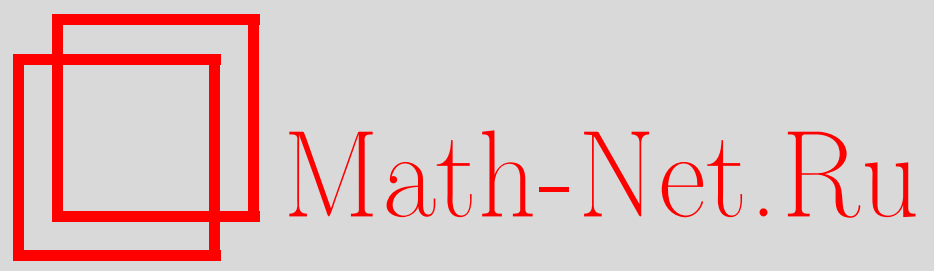

К. Н. Панков, Локальная предельная теорема для распределения части вектора весов подфункций компонент случайного двоичного отображения, Матем. вопр. криптогр., 2014, том 5, выпуск $3,49-80$

DOI: https://doi.org/10.4213/mvk129

Использование Общероссийского математического портала Math-Net.Ru подразумевает, что вы прочитали и согласны с пользовательским соглашением http://www . mathnet.ru/rus/agreement

Параметры загрузки:

IP : 54.197 .217 .227

26 апреля 2023 г., 13:34:16 
МАТЕМАТИЧЕСКИЕ ВОПРОСЫ КРИПТОГРАФИИ

2014 T. 5 № 3 C. $49-80$

УДК 519.212.2+519.214

\title{
Локальная предельная теорема для распределения части вектора весов подфункций компонент случайного двоичного отображения
}

\author{
К. Н. Панков
}

Московский государственный технический университет радиотехники, электроники и автоматики, г. Москва

Получено 22.IV.2013

Доказана локальная предельная теорема для распределения части вектора весов подфункций компонент (т. е. линейных комбинаций координатных функций) случайного двоичного отображения. С помощью этой теоремы найдены асимптотические оценки для числа $(n, m, k)$ устойчивых двоичных вектор-функций при $m=2,3,4$.

Ключевые слова: случайное двоичное отображение, локальная предельная теорема, веса подфункций, устойчивые булевы вектор-функции

Local limit theorem for the distribution of incomplete vector formed by the weights of subfunctions of random binary mapping components

K. N. Pankov

Moscow State Institute of Radio Engineering, Electronics and Automation, Moscow

Abstract. For linear combinations of coordinate functions of random binary mapping a local limit theorem for the joint distribution of weights of some their subfunctions is proved. By means of this theorem we obtain asymptotic estimates for the number of $(n, m, k)$-resilient vectorial Boolean functions for $m=2,3,4$.

Keywords: random binary mapping, local limit theorem, weights of subfunctions, resilient vectorial Boolean function

Citation: Mathematical Aspects of Cryptography, 2014, vol. 5, no. 3, pp. 49-80 (Russian)

(C) 2014 K. Н. Панков 
В [9], а затем в монографии [4] было доказано, что многие важные с точки зрения криптографии свойства двоичного отображения $f$, например, максимальная нелинейность, корреляционная иммунность, устойчивость, имеют место, если этими свойствами обладают все ненулевые линейные комбинации координатных функций $f$, называемых в [10] компонентными функииями или компонентами. Свойства же компонент могут быть, в частности, выражены в терминах весов подфункций.

Далее под обозначением $\psi_{m}(J)$, где $J=\left\{j_{1}, \ldots, j_{|J|}\right\}-$ подмножество множества $\overline{1, m}=\{1, \ldots, m\}$, мы будем понимать двоичный вектор из $V_{m}$ (множества двоичных векторов размерности $m$ ), у которого координаты с номерами $j_{1}, \ldots, j_{|J|}$ равны единице, а остальные - нулю, а под $(\bar{x}, \bar{y})=$ $x_{1} y_{1} \oplus \ldots \oplus x_{n} y_{n}-$ скалярное произведение векторов $\bar{x}$ и $\bar{y}$ из $V_{m}$.

Для произвольных подмножества $I=\left\{i_{1}, \ldots, i_{|I|}\right\}$ множества $\overline{1, n}$ и непустого подмножества $J$ множества $\overline{1, m}$ через $w_{I}^{J}(f)$ обозначим вес $\left\|\left(\psi_{m}(J), f\right)_{i_{1}, \ldots, i_{|I|}}^{1, \ldots, 1}\right\|$ подфункции $\left(\psi_{m}(J), f\right)_{i_{1}, \ldots, i_{|I|}}^{1, \ldots, 1}$ компоненты $\left(\psi_{m}(J), f\right)=f_{j_{1}} \oplus \ldots \oplus f_{j_{|J|}}$ отображения $f=\left(f_{1}, \ldots, f_{n}\right)$ из множества $B_{n}^{m}=\left\{f \mid f: V_{n} \rightarrow V_{m}\right\}$ всех $m$-мерных двоичных функций от $n$ переменных, получаемой, если у аргумента компоненты $\left(\psi_{m}(J), f\right)$ значения координат с номерами $i_{1}, \ldots, i_{|I|}$ положить равными единице.

Пусть функция $f$ выбирается случайно и равновероятно из множества $B_{n}^{m}$. Это эквивалентно независимому равновероятному выбору ее значений $f(\alpha)$ из множества $V_{m}$ двоичных векторов размерности $m$ для всех $\alpha$ из множества $V_{n}$. Можно рассматривать значение функции $f$ как вектор размерности $m$, состоящий из значений ее координатных двоичных функций от $n$ переменных:

$$
f(\alpha)=\left(f_{1}(\alpha), f_{2}(\alpha), \ldots, f_{m}(\alpha)\right): V_{n} \rightarrow V_{m}
$$

где $f_{i}(\alpha): V_{n} \rightarrow\{0,1\}$ для всех $i \in \overline{1, m}$.

Тогда значения $f_{i}(\alpha)$ выбираются независимо и равновероятно из множества $\{0,1\}$ для всех $\alpha$ из множества $V_{n}$ и для всех $i \in \overline{1, m}$.

При случайном выборе $f$ векторы, состоящие из части весов подфункций компонент отображения, также являются случайными, и возникает вопрос об их распределении. В [5] доказана асимптотическая нормальность векторов фиксированной размерности, состоящего из весов подфункций компонент случайной двоичной вектор-функции с оценкой скорости сходимости. 
В [3] доказана локальная предельная теорема для совместного распределения части весов подфункций случайной равновероятной функции (случай $m=1$ ). Однако в [11] отмечено, что в доказательстве этой теоремы имелась неточность, повлиявшая на формулировку теоремы. Будем использовать методы, предложенные в [3], исправив возникшую неточность и обобщив результаты на случай произвольного $m$.

Определим случайный вектор

$$
\bar{w}=\bar{w}(n, m, k)=\left(w^{J}, \varnothing \neq J \subset \overline{1, m}\right),
$$

где $w^{J}=\left(w_{I}^{J}(f), I \subset \overline{1, n}, 0 \leq|I| \leq k\right)$, размерности

$$
N(n, m, k)=\left(2^{m}-1\right) L(n, k), \text { где } L(n, k)=\sum_{i=0}^{k}\left(\begin{array}{c}
n \\
i
\end{array}\right) .
$$

Согласно следствию 1 [5] веса подфункций двоичного отображения удовлетворяют сравнению

$$
\sum_{\emptyset \neq S \subset J}(-1)^{|S|} w_{I}^{S} \equiv 0\left(\bmod 2^{|J|-1}\right) .
$$

Здесь и далее будем использовать обозначения $N=N(n, m, k)$, $Q=Q(n, m, k)$ - ковариационная матрица случайного вектора $(\bar{w}-\mathrm{E} \bar{w}) 2^{-n / 2-m+2}, \exp _{2} x=2^{x}$.

Теорема 1. Пусть $n \rightarrow \infty, k(n)=o(\sqrt{n}), k(n) \geq 1, m(n)=$ о $(n), \bar{z}(n) 2^{\frac{n}{2}+m-2}$ - такая последовательность иелочисленных векторстолбиов размерности $N(n, m, k)$, что координаты векторов из последовательности $\mathrm{E} \bar{w}+\bar{z} 2^{\frac{n}{2}+m-2}$ удовлетворяют сравнениям (1). Тогда равномерно относительно $\bar{z}(n)$ верно равенство

$$
\begin{aligned}
\mathrm{P}\left(\bar{w}=\mathrm{E} \bar{w}+\bar{z} 2^{\frac{n}{2}+m-2}\right)=\frac{\exp \left(-\frac{1}{2} z^{T} Q^{-1} z\right)+O\left(n^{3 k+3} 2^{3 m-\frac{n}{2}}\right)}{2^{\frac{n+2 m-3}{2} N} \sqrt{\pi^{N} \operatorname{det} Q}} \times \\
\quad \times\left(\sum_{\vec{r} \in \mathfrak{R}^{* *}(m, N)} \exp \left[-i \pi 2^{n / 2}(\vec{r}, z)\right]\right)=
\end{aligned}
$$




$$
=\frac{\exp \left(-2^{2 m-3} \sum_{\varnothing \neq J \subset \overline{1, m}} \sum_{\substack{I \subset \overline{1, n} \\
|I| \leq k}}\left(\sum_{K \subset I}(-1)^{|K|} 2^{|K|} z_{K}^{J}\right)^{2}\right)+O\left(n^{3 k+3} 2^{3 m-\frac{n}{2}}\right)}{\exp _{2}\left(\frac{(n-k)}{2}\left(\begin{array}{c}
n \\
k
\end{array}\right)\left(2^{m}-1\right)+N(n, m, k) \log _{2} \sqrt{\pi / 2}\right)} \times
$$

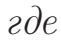

$$
\begin{gathered}
\mathfrak{R}^{* *}(m, N)=\left\{\vec{r} \in\left\{0, \ldots, 2^{m-1}-1\right\}^{N}: \forall I, \forall s \in \overline{1, m}, \forall \delta \in V_{m}\right. \\
\left.\sum_{J \subset \overline{1, m}, s \in J}(-1)^{\left(\delta, \psi_{m}(J)\right) \oplus 1} r_{I}^{J} \in 2^{m-1} \mathbb{Z}\right\} .
\end{gathered}
$$

Доказательство. Очевидно, что $\mathrm{E} w_{I}^{J}=2^{n-|I|-1}$ и выполняется следующее равенство:

$$
\begin{gathered}
\frac{w_{I}^{J}-\mathrm{E} w_{I}^{J}}{2^{m-1}}=\sum_{\alpha \in V_{n}} \beta_{I}(\alpha)\left(\frac{2\left(\psi_{m}(J), f\right)(\alpha)-1}{2^{m}}\right)= \\
=2^{-m} \sum_{\alpha \in V_{n}} \beta_{I}(\alpha)(-1)^{\left(\psi_{m}(J), f\right)(\alpha) \oplus 1},
\end{gathered}
$$

где $\beta_{I}(\alpha)=\alpha_{i_{1}} \alpha_{i_{2}} \ldots \alpha_{i_{|I|}}$ (при $I=\varnothing$ считаем, что $\left.\beta_{I}(\alpha) \equiv 1\right)$ ).

Тогда для векторов

$$
\begin{gathered}
\beta^{k}(\alpha)=\left(\beta_{I}(\alpha), I \subset \overline{1, n}, 0 \leq|I| \leq k\right) \\
\xi(\alpha)=\left(\beta^{k}(\alpha)\left(\frac{2\left(\psi_{m}(J), f\right)-1}{2^{m}}\right), \varnothing \neq J \subset \overline{1, m}\right)= \\
=\left(2^{-m}(-1)^{\left(\psi_{m}(J), f\right)(\alpha) \oplus 1} \beta^{k}(\alpha), \varnothing \neq J \subset \overline{1, m}\right)
\end{gathered}
$$

и $\xi=2^{1-m}(\bar{w}-\mathrm{E} \bar{w})$ справедливо соотношение $\xi=\sum_{\alpha \in V_{n}} \xi(\alpha)$.

Характеристические функции случайных векторов $\xi(\alpha)$ и $\xi$ равны соответственно

$$
\phi_{\alpha}(t)=\sum_{\delta \in V_{m}} \frac{1}{2^{m}} \exp \left[i\left(\beta^{k}(\alpha), \sum_{\varnothing \neq J \subset \overline{1, m}}(-1)^{\left(\delta, \psi_{m}(J)\right) \oplus 1} 2^{-m} t^{J}\right)\right],
$$




$$
\varphi_{\xi}(t)=\prod_{\alpha \in V_{n}} \varphi_{\alpha}(t)
$$

где $\delta=\left(\delta_{1}, \ldots, \delta_{m}\right), \delta_{i} \in\{0,1\}, t \in \mathbb{R}^{N}, t=\left(t^{J}, \varnothing \neq J \subset \overrightarrow{1, m}\right), t^{J}=$ $\left(t_{I}^{J}, 0 \leq|I| \leq k\right), t^{J} \in \mathbb{R}^{L(n, k)}$.

Умножив равенство $\varphi_{\xi}(t)=\sum_{b \in \mathbb{Z}^{N}} \mathrm{P}(\xi=b) \exp [i(t, b)]$ на выражение $\exp \left[-i\left(t, z 2^{n / 2-1}\right)\right]$ и проинтегрировав его по переменным $t_{I}^{J}$ в пределах от $-\pi$ до $2^{m} \pi-\pi$, находим, что

$$
\left(2^{m} \pi\right)^{N} \mathrm{P}\left(\xi=z 2^{n / 2-1}\right)=\int_{-\pi}^{2^{m} \pi-\pi} \cdots \int_{-\pi}^{2^{m} \pi-\pi} \phi_{\xi}(t) \exp \left[-i\left(t, z 2^{n / 2-1}\right)\right] d t .
$$

Представим интеграл из левой части данного равенства по области $G=$ $\left[-\pi, 2^{m} \pi-\pi\right]^{N}$, используя разбиение отрезка

$$
\begin{aligned}
{\left[-\pi, 2^{m} \pi-\pi\right]=[-\pi, \pi] \cup[\pi, 3 \pi] \cup \ldots \cup } & {\left[2^{m} \pi-3 \pi, 2^{m} \pi-\pi\right]=} \\
& =\bigcup_{r=0}^{2^{m-1}-1}[-\pi+2 \pi r, \pi+2 \pi r]
\end{aligned}
$$

как сумму интегралов по областям

$$
\begin{aligned}
G(\vec{r})=G\left(r_{I}^{J}, \varnothing \neq J \subset \overline{1, m}, I \subset \overline{1, n},|I| \leq k\right)= & \\
& =\left\{t \in G:-\pi+2 \pi r_{I}^{J} \leq t_{I}^{J} \leq \pi+2 \pi r_{I}^{J}\right\},
\end{aligned}
$$

где $\vec{r} \in R(m, N)=\left\{0,1, \ldots, 2^{m-1}-1\right\}^{N}$.

Получаем

$$
\left(2^{m} \pi\right)^{N} \mathrm{P}\left(\xi=z 2^{\frac{n}{2}-1}\right)=\sum_{\vec{r} \in R(m, N)} \int_{G(\vec{r})} \phi_{\xi}(t) \exp \left[-i\left(t, z 2^{\frac{n}{2}-1}\right)\right] d t .
$$

Производя замену $x=t 2^{n / 2-1}$ и обозначая через $H(\vec{r})$ области

$$
\left\{x \in 2^{n / 2-1} G:-\pi 2^{-1+n / 2}+2^{n / 2} \pi r_{I}^{J} \leq x_{I}^{J} \leq \pi 2^{-1+n / 2}+2^{n / 2} \pi r_{I}^{J}\right\}
$$

мы приходим к равенству

$$
\left(2^{m-1+\frac{n}{2}} \pi\right)^{N} \mathrm{P}\left(\xi=z 2^{-1+\frac{n}{2}}\right)=\sum_{\vec{r} \in R(m, N)} \int_{H(\vec{r})} \phi_{\xi}\left(x 2^{1-\frac{n}{2}}\right) \exp [-i(x, z)] d x
$$




$$
\phi_{\xi}\left(x 2^{1-n / 2}\right)=\prod_{\alpha \in V_{n}} \phi_{\alpha}\left(x 2^{1-n / 2}\right)
$$

Для всех $t \in\{0,1, \ldots, k\}$ определим функцию $\Lambda(n, k, t)=\left(\left(\begin{array}{c}n \\ t\end{array}\right) 2^{\frac{5 n}{12}+k t}\right)^{-1}$ и разобьем все $H(\vec{r})$ на попарно не пересекающиеся области

$$
\begin{gathered}
H_{t}(\vec{r})=\left\{x \in H(\vec{r}), \exists J, I:|I|=t,\left|\frac{x_{I}^{J}}{2^{n / 2}}-\pi r_{I}^{J}\right|>\Lambda(n, k, t) ;\right. \\
\left.\forall L, K:|K|>t,\left|\frac{x_{K}^{L}}{2^{n / 2}}-\pi r_{K}^{L}\right| \leq \Lambda(n, k,|K|)\right\}, \\
H^{*}(\vec{r})=H(\vec{r}) \backslash\left(\bigcup_{t=0}^{k} H_{t}(\vec{r})\right)= \\
\left\{x \in G, \forall J, I:\left|\frac{x_{I}^{J}}{2^{n / 2}}-\pi r_{I}^{J}\right| \leq \Lambda(n, k,|I|)\right\} .
\end{gathered}
$$

Разобьем каждый интеграл $\int_{H(\vec{r})} \phi_{\xi}\left(x 2^{1-n / 2}\right) \exp [-i(x, z)] d t$ на сумму интегралов по областям $H(\vec{r}) \backslash H^{*}(\vec{r})$ и $H^{*}(\vec{r})$, и обозначим их соответственно $J_{1}(\vec{r})$ и $J_{2}(\vec{r})$

Дальнейшее доказательство проведем поэтапно. В следующей лемме и везде далее все неравенства выполняются, начиная с некоторого $n_{0}$.

Оценим интегралы $J_{1}(\vec{r})$.

Лемма 2. Для любого $\vec{r} \in R(m, N)$ верно неравенство

$$
\left|J_{1}(\vec{r})\right| \leq\left(\left(2^{n / 2} \pi\right)^{N}-V\left(H^{*}(\vec{r})\right)\right) \exp \left(-2^{n / 7}\right),
$$

где $V\left(H^{*}(\vec{r})\right)$ - мера Лебега области $H^{*}(\vec{r})$.

Доказательство. Если $x \in H_{t}(\vec{r})$ для некоторого $t \in \overline{0, k}$ и некоторого вектора из $N$ целых чисел $r_{I}^{J}$, то существуют такие множества $S, I$, что $\varnothing \neq S \subset \overline{1, m},|I|=t,\left|x_{I}^{S} 2^{-n / 2}-\pi r_{I}^{S}\right|>\Lambda(n, k, t)$, а для любых таких множеств $L, K$, что $|K|>t$, выполняется $\left|x_{K}^{L} 2^{-n / 2}-\pi r_{K}^{L}\right| \leq \Lambda(n, k,|K|)$

Без ограничения общности можно считать, что $S=$ $\{m-s+1, \ldots, m\}$, где $s \leq m, I=\{1, \ldots, t\}$. Рассмотрим харак- 
теристическую функцию

$$
\begin{aligned}
& \phi_{\alpha}\left(x 2^{1-\frac{n}{2}}\right)= \\
& \sum_{\delta \in V_{m}} \frac{1}{2^{m}} \exp \left[i\left(\left(\beta^{k}(\alpha), \sum_{\varnothing \neq J \subset \overline{1, m},|J| \leq k}(-1)^{\left(\delta, \psi_{m}(J)\right) \oplus 1} 2^{1-m-n / 2} x^{J}\right)\right] .\right.
\end{aligned}
$$

Обозначим $y=2^{1-n / 2-m} x, y^{*}=2^{1-n / 2-m} x-2^{1-m} \pi \cdot \vec{r}$. Тогда выполняются неравенства $\left|y_{I}^{S}-2^{1-m} \pi r_{I}^{S}\right|>2^{1-m} \Lambda(n, k, t),\left|y_{I}^{S} *\right|>2^{1-m} \Lambda(n, k, t)$, а для любых таких $L, K$, что $|K|>t$, выполняются неравенства $\left|y_{K}^{L}-2^{1-m} \pi r_{K}^{L}\right| \leq 2^{1-m} \Lambda(n, k,|K|),\left|y_{K}^{L *}\right| \leq 2^{1-m} \Lambda(n, k,|K|)$.

Тогда мы можем переписать равенство для характеристической функции в виде

$$
\begin{aligned}
& \varphi_{\alpha}\left(2^{m} y\right)=\sum_{\delta \in V_{m}} \frac{1}{2^{m}} \exp \left[i\left(\beta^{k}(\alpha), \sum_{\varnothing \neq J \subset \overline{1, m}}(-1)^{\left(\delta, \psi_{m}(J)\right) \oplus 1} y^{J}\right)\right]= \\
& =\sum_{\left(\delta_{1}, \ldots, \delta_{m-1}\right)=\delta^{\prime} \in V_{m-1}} \frac{1}{2^{m-1}} \exp \left[i\left(\beta^{k}(\alpha), \sum_{\varnothing \neq J^{\prime} \subset \overline{1, m-1}}(-1)^{\left(\delta^{\prime}, \psi_{m-1}\left(J^{\prime}\right)\right) \oplus 1} y^{J}\right)\right] \times \\
& \times \sum_{\delta_{m}=0}^{1} \frac{1}{2} \exp \left[i\left(\beta^{k}(\alpha), \sum_{J^{\prime \prime} \subset \overline{1, m-1}}(-1)^{\left(\delta^{\prime}, \psi_{m-1}\left(J^{\prime \prime}\right)\right) \oplus \delta_{m} \oplus 1} y^{J^{\prime \prime} \cup\{m\}}\right)\right]= \\
& =\sum_{\left(\delta_{1}, \ldots, \delta_{m-1}\right)=\delta^{\prime} \in V_{m-1}} \frac{1}{2^{m-1}} \exp \left[i\left(\beta^{k}(\alpha), \sum_{\varnothing \neq J \subset \overline{1, m-1}}(-1)^{\left(\delta^{\prime}, \psi_{m-1}(J)\right) \oplus 1} y^{J}\right)\right] \times \\
& \times \cos \left(\beta^{k}(\alpha), \sum_{J \subset \overline{1, m-1}}(-1)^{\left(\delta^{\prime}, \psi_{m}(J)\right)} y^{J \cup\{m\}}\right) .
\end{aligned}
$$

Очевидно, что

$$
\left|\varphi_{\alpha}\left(2^{m} y\right)\right| \leq \sum_{\delta \in V_{m-1}} \frac{1}{2^{m-1}}\left|\cos \left(\beta^{k}(\alpha), \sum_{J \subset \overline{1, m-1}}(-1)^{\left(\delta, \psi_{m-1}(J)\right)} y^{J \cup\{m\}}\right)\right|,
$$


где

$$
\begin{aligned}
& \left(\beta^{k}(\alpha), \sum_{J \subset \overline{1, m-1}}(-1)^{\left(\delta, \psi_{m-1}(J)\right)} y^{J \cup\{m\}}\right)= \\
& =\left(\beta^{k}(\alpha),(-1)^{\left(\delta, \psi_{m-1}(S \backslash\{m\})\right)} y^{S}\right)+\left(\beta^{k}(\alpha), \sum_{\substack{J \subset \overline{1, m-1} \\
J \neq S \backslash\{m\}}}(-1)^{\left(\delta, \psi_{m-1}(J)\right)} y^{J \cup\{m\}}\right) .
\end{aligned}
$$

Следовательно,

$$
\begin{aligned}
\left|\varphi_{\alpha}\left(2^{m} y\right)\right| \leq \sum_{\delta \in V_{m-1}} \frac{1}{2^{m-1}} \mid \cos \left(\left(\beta^{k}(\alpha), y^{S}\right)+\right. \\
\left.\quad\left(\beta^{k}(\alpha), \sum_{J \subset \overline{1, m-1, J \neq S \backslash\{m\}}}(-1)^{\left(\delta, \psi_{m-1}(J)\right) \oplus\left(\delta, \psi_{m-1}(S \backslash\{m\})\right)} y^{J \cup\{m\})}\right)\right) .
\end{aligned}
$$

Для всех $\delta \in V_{m-1}$ обозначим

$$
\begin{array}{r}
\left(\beta^{k}(\alpha), y^{S}\right)+\left(\beta^{k}(\alpha), \sum_{\substack{J \subset \overline{1, m-1} \\
J \neq S \backslash\{m\}}}(-1)^{\left(\delta, \psi_{m-1}(J)\right) \oplus\left(\delta, \psi_{m-1}(S \backslash\{m\})\right)} y^{J \cup\{m\}}\right)= \\
=\rho^{\delta}(\alpha)+\pi l^{\delta}(\alpha),
\end{array}
$$

где $\left|\rho^{\delta}(\alpha)\right| \leq \pi / 2, l^{\delta}(\alpha) \in Z$.

Так как $\cos \rho^{\delta}(\alpha) \leq 1-\left(\rho^{\delta}(\alpha) / 2\right)^{2}$ при всех $\left|\rho^{\delta}(\alpha)\right| \leq \pi / 2$, то

$$
\left|\phi_{\alpha}\left(2^{m} y\right)\right| \leq \sum_{\delta \in V_{m-1}} \frac{\cos \rho^{\delta}(\alpha)}{2^{m-1}} \leq 1-2^{-m-1} \max _{\delta \in V_{m-1}}\left(\rho^{\delta}(\alpha)\right)^{2} .
$$

Разобьем $V_{n}$ на $2^{n-t}$ групп векторов с одинаковыми последними $n-t$ координатами. Покажем, что в группе с произвольным вектором $\gamma$ последних $n-t$ координат найдется вектор $\alpha$, для которого существует такой $\delta \in V_{m-1}$, что $\left|\rho^{\delta}(\alpha)\right| \geq 2^{1-k-t-m} \Lambda(n, k, t)$. Для этого рассмотрим 
величины

$$
\begin{gathered}
W_{0}^{\delta}\left(\alpha_{1}, \ldots, \alpha_{t}\right)=\left(\beta^{k}\left(\alpha_{1}, \ldots, \alpha_{t}, \gamma\right), y^{S}\right)+ \\
+\left(\beta^{k}\left(\alpha_{1}, \ldots, \alpha_{t}, \gamma\right), \sum_{J \subset \overline{1, m-1}, J \neq S \backslash\{m\}}(-1)^{\left(\delta, \psi_{m-1}(J)\right) \oplus\left(\delta, \psi_{m-1}(S \backslash\{m\})\right)} y J\{m\}\right) \\
=\rho_{0}^{\delta}\left(\alpha_{1}, \ldots, \alpha_{t}\right)+\pi l_{0}^{\delta}\left(\alpha_{1}, \ldots, \alpha_{t}\right), \\
W_{0}\left(\alpha_{1}, \ldots, \alpha_{t}\right)=\sum_{\delta \in V_{m-1}} W_{0}^{\delta}\left(\alpha_{1}, \ldots, \alpha_{t}\right)=\rho_{0}\left(\alpha_{1}, \ldots, \alpha_{t}\right)+\pi l_{0}\left(\alpha_{1}, \ldots, \alpha_{t}\right), \\
W_{1}\left(\alpha_{2}, \ldots, \alpha_{t}\right)=W_{0}\left(1, \alpha_{2}, \ldots, \alpha_{t}\right)-W_{0}\left(0, \alpha_{2}, \ldots, \alpha_{t}\right)= \\
\quad=\rho_{1}\left(\alpha_{2}, \ldots, \alpha_{t}\right)+\pi l_{1}\left(\alpha_{2}, \ldots, \alpha_{t}\right), \\
W_{t}(\varnothing)=W_{t-1}(1)-W_{t-1}(0)=\rho_{t}(\varnothing)+\pi l_{t}(\varnothing)
\end{gathered}
$$

где модули величин $\left|\rho_{0}^{\delta}\left(\alpha_{1}, \ldots, \alpha_{t}\right)\right|,\left|\rho_{0}\left(\alpha_{1}, \ldots, \alpha_{t}\right)\right|, \ldots,\left|\rho_{t}(\varnothing)\right|$ не превосходят $\pi / 2$, а величины $l_{0}^{\delta}\left(\alpha_{1}, \ldots, \alpha_{t}\right), l_{0}\left(\alpha_{1}, \ldots, \alpha_{t}\right), \ldots, l_{t}(\varnothing)$ являются целыми.

Предположим противное. Пусть $\left|\rho_{0}^{\delta}\left(\alpha_{1}, \ldots, \alpha_{t}\right)\right|<2^{1-k-t-m} \Lambda(n, k, t)$ для всех величин $\alpha_{1}, \ldots, \alpha_{t}$.

Тогда $\rho_{i}(\varnothing)$, как линейная комбинация $2^{m-1+t}$ величин $\rho_{0}^{\delta}\left(\alpha_{1}, \ldots, \alpha_{t}\right)$ с коэффициентами \pm 1 , имеет ограничение $\left|\rho_{t}(\varnothing)\right|<2^{-k} \Lambda(n, k, t)$.

С другой стороны,

$$
\begin{aligned}
& W_{0}\left(\alpha_{1}, \ldots, \alpha_{t}\right)=\sum_{\delta \in V_{m-1}}\left(\beta^{k}\left(\alpha_{1}, \ldots, \alpha_{t}, \gamma\right), y^{S}\right)+ \\
& +\sum_{\delta \in V_{m-1}}\left(\beta^{k}\left(\alpha_{1}, \ldots, \alpha_{t}, \gamma\right), \sum_{J \subset \overline{1, m-1}, J \neq S \backslash\{m\}}(-1)^{\left(\delta, \psi_{m-1}(J)\right) \oplus\left(\delta, \psi_{m-1}(S \backslash\{m\})\right)} y^{J \cup\{m\}}\right)= \\
& =2^{m-1}\left(\beta^{k}\left(\alpha_{1}, \ldots, \alpha_{t}, \gamma\right), y^{S}\right)+ \\
& +\sum_{J \subset \overline{1, m-1, J \neq S \backslash\{m\}}}\left(\beta^{k}\left(\alpha_{1}, \ldots, \alpha_{t}, \gamma\right), y^{J \cup\{m\}}\right) \sum_{\delta \in V_{m-1}}(-1)^{\left(\delta, \psi_{m-1}(J)\right) \oplus\left(\delta, \psi_{m-1}(S \backslash\{m\})\right)} .
\end{aligned}
$$

Если $J \neq S \backslash\{m\}$, то очевидно, что $\psi_{m-1}(J) \oplus \psi_{m-1}(S \backslash\{m\}) \neq \overline{0}$ и верно равенство $\sum_{\delta \in V_{m-1}}(-1)^{\left(\delta, \psi_{m-1}(J)\right) \oplus\left(\delta, \psi_{m-1}(S \backslash\{m\})\right)}=0$. 
Следовательно,

$$
\begin{aligned}
W_{0}\left(\alpha_{1}, \ldots, \alpha_{t}\right)=2^{m-1}\left(\beta^{k}\left(\alpha_{1}, \ldots, \alpha_{t}, \gamma\right), y^{S}\right)= & \\
& =2^{m-1} \sum_{I \subset \overline{1, n},|I| \leq k} \beta_{I}\left(\alpha_{1}, \ldots, \alpha_{t}, \gamma\right) \cdot y_{I}^{S},
\end{aligned}
$$

$W_{1}\left(\alpha_{2}, \ldots, \alpha_{t}\right)=$

$$
\begin{array}{r}
=2^{m-1} \sum_{I \subset \overline{1, n},|I| \leq k}\left(\beta_{I}\left(1, \alpha_{2}, \ldots, \alpha_{t}, \gamma\right)-\beta_{I}\left(0, \alpha_{2}, \ldots, \alpha_{t}, \gamma\right)\right) y_{I}^{S}= \\
=\sum_{I \subset \overline{1, n}, I \supset\{1\},|I| \leq k} \beta_{I}\left(1, \alpha_{2}, \ldots, \alpha_{t}, \gamma\right) \cdot y_{I}^{S},
\end{array}
$$

$$
\begin{gathered}
W_{t}(\varnothing)=2^{m-1} \sum_{I \subset \overline{1, n}, I \supset\{1, \ldots, t\}} \beta_{I}(1, \ldots, 1, \gamma) \cdot y_{I}^{S}=2^{m-1} y_{\{1, \ldots, t\}}^{S}+ \\
+2^{m-1} \sum_{I \subset \overline{1, n}, I \supset\{1, \ldots, t\}, t<|I| \leq k>t} \beta_{I}(1, \ldots, 1, \gamma) \cdot y_{I}^{S}= \\
=2^{m-1} y_{I}^{S *}+\pi r_{I}^{S}+2^{m-1} \sum_{I \subset \overline{1, n}, I \supset\{1, \ldots, t\}, t<|I| \leq k} \beta_{I}(1, \ldots, 1, \gamma) \cdot y_{K}^{S *}+ \\
+\sum_{I \subset \overline{1, n}, I \supset\{1, \ldots, t\}, t<|I| \leq k} \beta_{I}(1, \ldots, 1, \gamma) \cdot \pi r_{K}^{S}= \\
=C^{\prime} \pi+2^{m-1} y_{I}^{S *}+2^{m-1} \sum_{I \subset \overline{1, n}, I \supset\{1, \ldots, t\}, t<|I| \leq k} \beta_{I}(1, \ldots, 1, \gamma) \cdot y_{K}^{S *},
\end{gathered}
$$

где $C^{\prime}=r_{I}^{S}+\sum_{I \subset \overline{1, n}, I \supset\{1, \ldots, t\}, t<|I| \leq k} \beta_{I}(1, \ldots, 1, \gamma) r_{K}^{S} \in \mathbb{Z}$.

Поскольку $2^{1-m} \Lambda(n, k, t)<\left|y_{I}^{S^{*}}\right| \leq \pi / 2$ и модуль последней суммы не 
превосходит величины

$$
\begin{gathered}
2^{m-1} \sum_{j=1}^{k-t}\left(\begin{array}{c}
n-t \\
j
\end{array}\right) \cdot 2^{1-m}\left[\left(\begin{array}{c}
n \\
t+j
\end{array}\right) 2^{-k(t+j)-5 n / 12}\right]^{-1} \leq \\
\leq 2^{-k t-5 n / 12}\left[\left(\begin{array}{l}
n \\
t
\end{array}\right)\right]^{-1} \cdot 2^{-k} \sum_{j=1}^{k-t}\left(\begin{array}{c}
t+j \\
j
\end{array}\right) \leq \\
\leq \Lambda(n, k, t) \cdot 2^{-k} \sum_{j=1}^{k}\left(\begin{array}{c}
k \\
j
\end{array}\right)=\Lambda(n, k, t) \cdot 2^{-k}\left(2^{k}-1\right)=\Lambda(n, k, t) \cdot\left(1-2^{-k}\right)
\end{gathered}
$$

то справедлива оценка

$$
\begin{aligned}
\Lambda(n, k, t)-\Lambda(n, k, t) \cdot\left(1-2^{-k}\right) \leq\left|W_{t}(\varnothing)-C^{\prime} \pi\right| \leq \\
\leq \pi / 2+\Lambda(n, k, t) \cdot\left(1-2^{-k}\right) \leq \pi-2^{-k} \Lambda(n, k t) .
\end{aligned}
$$

Следовательно, $\left|\rho_{t}(\varnothing)\right|>2^{-k} \Lambda(n, k, t)$, что противоречит оценке, полученной из предположения.

Из данного факта получаем, что для любого $x \in H_{t}$ не менее чем для $2^{n-t}$ векторов $\alpha$ из $V_{n}$ выполняется неравенство

$$
\left|\varphi_{\alpha}\left(x 2^{1-n / 2}\right)\right| \leq 1-2^{-m-1}\left(2^{1-k-t-m} \Lambda(n, k, t)\right)^{2}=1-\frac{(\Lambda(n, k, t))^{2}}{2^{2 k+2 t+3 m-1}} .
$$

Следовательно, для любого $x \in H_{t}(\vec{r})$ :

$$
\begin{aligned}
\left|\phi_{\xi}\left(x 2^{1-n / 2}\right)\right|=\left|\prod_{\alpha \in V_{n}} \phi_{\alpha}\left(x 2^{1-n / 2}\right)\right| \leq\left(1-\frac{(\Lambda(n, k, t))^{2}}{2^{2 k+2 t+3 m-1}}\right)^{2^{n-t}} \leq \\
\leq \exp \left(-2^{n-t} \frac{(\Lambda(n, k, t))^{2}}{2^{2 k+2 t+3 m-1}}\right)=\exp \left(-\frac{2^{n-3 m-3 t-2 k+1}}{\left(\begin{array}{l}
n \\
t
\end{array}\right)^{2} 2^{2 k t+5 n / 6}}\right) \leq \\
\leq \exp \left(-\frac{2^{n / 6-2 k^{2}-3 m-5 k+1}}{\left(\begin{array}{l}
n \\
k
\end{array}\right)^{2}}\right) .
\end{aligned}
$$

Так как $k^{2}=o(n), m(n)=o(n)$, то для любого $x \in H(\vec{r}) \backslash H^{*}(\vec{r})$ справедливо неравенство $\left|\phi_{\xi}\left(x 2^{1-n / 2}\right)\right| \leq \exp \left(-2^{n / 7}\right)$, и верна следую- 
щая оценка:

$$
\begin{aligned}
& \left|J_{1}(\vec{r})\right|=\left|\int_{H(\vec{r}) \backslash H^{*}(\vec{r})} \phi_{\xi}\left(x 2^{1-n / 2}\right) \exp [-i(x, z)] d x\right| \leq \\
& \leq \exp \left(-2^{n / 7}\right)\left|\int_{H(\vec{r}) \backslash H^{*}(\vec{r})} d x\right|=\left(\left(2^{n / 2} \pi\right)^{N}-V\left(H^{*}(\vec{r})\right)\right) \exp \left(-2^{n / 7}\right) .
\end{aligned}
$$

Теперь рассмотрим поведение $\phi_{\xi}\left(x 2^{1-n / 2}\right)$ в областях $H^{*}(\vec{r})$. Для всех $x \in H^{*}(\vec{r})$ верно неравенство $\left|x_{I}^{J}-\pi 2^{n / 2} r_{I}^{J}\right| \leq 2^{n / 2} \Lambda(n, k,|I|)$. Обозначим $x^{*}=2^{1-n / 2-m} x-2^{1-m} \pi \cdot \vec{r}$. Тогда выполняется неравенство $\left|x_{I}^{J^{*}}\right| \leq \frac{\Lambda(n, k,|I|)}{2^{m-1}}$.

Рассмотрим характеристическую функцию:

$$
\begin{aligned}
& \phi_{\alpha}\left(x 2^{1-n / 2}\right)= \\
& =\sum_{\delta \in V_{m}} \frac{1}{2^{m}} \exp \left[i\left(\left(\beta^{k}(\alpha), \sum_{\varnothing \neq J \in \overline{1, m}}(-1)^{\left(\delta, \psi_{m}(J) \oplus \oplus 1\right.} 2^{1-n / 2-m} x^{J}\right)\right]=\right. \\
& =\sum_{\delta \in V_{m}} \frac{1}{2^{m}} \exp \left[i\left(\sum_{\varnothing \neq J \subset \overline{1, m}}(-1)^{\left(\delta, \psi_{m}(J)\right) \oplus 1} \cdot 2^{1-n / 2-m} \sum_{I \subset \overline{1, n},|I| \leq k} \beta_{I}^{J}(\alpha) x_{I}^{J}\right)\right]= \\
& =\sum_{\delta \in V_{m}} \frac{1}{2^{m}} \exp \left[i\left(\sum_{\varnothing \neq J \subset \overline{1, m}}(-1)^{\left.\left(\delta, \psi_{m}(J)\right) \oplus 1 \sum_{I \subset \overline{1, n},|I| \leq k} \beta_{I}^{J}(\alpha)\left(x_{I}^{J *}+2^{1-m} \pi r_{I}^{J}\right)\right]}\right]\right.
\end{aligned}
$$

Если обозначить $d_{\alpha}^{J}=\left(\beta^{k}(\alpha), x^{J *}\right)$, то очевидно, что

$$
\left|d_{\alpha}^{J}\right| \leq \sum_{t=0}^{k-1}\left(\begin{array}{l}
n \\
t
\end{array}\right) \frac{\Lambda(n, k, t)}{2^{m-1}}=2^{1-5 n / 12-m} \sum_{t=0}^{k-1} 2^{-k t} \leq 2^{2-5 n / 12-m}
$$


и характеристическая функция будет иметь вид:

$$
\begin{aligned}
& \phi_{\alpha}\left(x 2^{1-n / 2}\right)= \\
&=\sum_{\delta \in V_{m}} \frac{1}{2^{m}} \exp \left[i\left(\sum_{\varnothing \neq J \subset \overline{1, m}}(-1)^{\left(\delta, \psi_{m}(J)\right) \oplus 1}\left(d_{\alpha}^{J}+2^{1-m} \pi \sum_{I \subset \overline{1, n},|I| \leq k} \beta_{I}^{J}(\alpha) r_{I}^{J}\right)\right)\right]= \\
&=\sum_{\delta \in V_{m}} \frac{1}{2^{m}} \exp \left[i \left(\sum_{\varnothing \neq J \subset \overline{1, m}}(-1)^{\left(\delta, \psi_{m}(J)\right) \oplus 1} d_{\alpha}^{J}+\right.\right. \\
&+2^{1-m} \sum_{\left.\left.\left(\beta^{k}(\alpha), \sum_{\varnothing \neq J \subset \overline{1, m}}(-1)^{\left(\delta, \psi_{m}(J)\right) \oplus 1} r^{J}\right)\right)\right]}
\end{aligned}
$$

Тогда

$$
\begin{array}{r}
\phi_{\alpha}\left(x 2^{1-\frac{n}{2}}\right)=\sum_{\delta \in V_{m}} \frac{1}{2^{m}} \exp \left[2^{1-m} \pi i\left(\beta^{k}(\alpha), \sum_{\varnothing \neq J \subset \overline{1, m}}(-1)^{\left(\delta, \psi_{m}(J)\right) \oplus 1} r^{J}\right)\right] \times \\
\times \exp \left[i \sum_{\varnothing \neq J \subset \overline{1, m},|J| \leq k}(-1)^{\left(\delta, \psi_{m}(J)\right) \oplus 1} d_{\alpha}^{J}\right] .
\end{array}
$$

Согласно лемме 1 [7, с. 131] для любых вещественных $x$ верно представление $\exp i x=1+\theta_{1}(x) \cdot x$, где $\left|\theta_{1}(x)\right| \leq 1$. Очевидно, что

$$
\left|\sum_{\varnothing \neq J \subset \overline{1, m},|J| \leq k}(-1)^{\left(\delta, \psi_{m}(J)\right) \oplus 1} d_{\alpha}^{J}\right| \leq 2^{2-5 n / 12} .
$$

Следовательно,

$$
\begin{aligned}
& \phi_{\alpha}\left(x 2^{1-n / 2}\right)= \\
& =\sum_{\delta \in V_{m}} \frac{1}{2^{m}} \exp \left[2^{1-m} \pi i\left(\beta^{k}(\alpha), \sum_{\varnothing \neq J \subset \overline{1, m}}(-1)^{\left(\delta, \psi_{m}(J)\right) \oplus 1} r^{J}\right)\right]+O\left(2^{-5 n / 12}\right) .
\end{aligned}
$$

Теперь разобьем множество $R(m, N)=\left\{0,1, \ldots, 2^{m-1}-1\right\}^{N}$ на попарно 
не пересекающиеся подмножества по всем $t \in \overline{0, k}$ :

$$
\begin{aligned}
& R_{t}(m, N)=\{\vec{r} \in R(m, N): \\
& \exists I,|I|=t, \exists s \in \overline{1, m}, \exists \delta \in V_{m} \sum_{J \subset \overline{1, m}, s \in J}(-1)^{\left(\delta, \psi_{m}(J)\right) \oplus 1} r_{I}^{J} \cdot 2^{1-m} \notin \mathbb{Z} \\
& \left.\forall K,|K|>t, \forall q \in \overline{1, m}, \forall \gamma \in V_{m} \sum_{L \subset \overline{1, m}, q \in L}(-1)^{\left(\gamma, \psi_{m}(L)\right) \oplus 1} r_{K}^{L} \cdot 2^{1-m} \in \mathbb{Z}\right\}, \\
& \mathfrak{R}^{* *}(m, N)=\left\{\vec{r} \in R(m, N): \forall I, \forall s \in \overline{1, m}, \forall \delta \in V_{m}\right. \\
& \left.\sum_{J \subset \overline{1, m}, s \in J}(-1)^{\left(\delta, \psi_{m}(J)\right) \oplus 1} r_{I}^{J} \cdot 2^{1-m} \in \mathbb{Z}\right\} .
\end{aligned}
$$

Лемма 3. Для всех $\vec{r} \in R_{t}(m, N)$

$$
\left|J_{2}(\vec{r})\right| \leq V\left(H^{*}(\vec{r})\right) \exp \left(-2^{n / 7}\right)
$$

где $V\left(H^{*}(\vec{r})\right)$ - мера Лебега области $H^{*}(\vec{r})$.

Доказательство. Пусть $\vec{r} \in R_{t}(m, N)$, тогда существуют такие множество $I,|I|=t$, натуральное число $s \in \overline{1, m}$ и вектор $\delta^{\prime} \in V_{m}$, что $\sum_{J \subset 1, m, s \in J}(-1)^{\left(\delta^{\prime}, \psi_{m}(J)\right) \oplus 1} r_{I}^{J} \cdot 2^{1-m} \notin \mathbb{Z}$, а для любых множеств $K$ с $|K|>t$ при любых $q \in \overline{1, m}, \gamma \in V_{m}$ сумма $\sum_{L \subset 1, m, q \in L}(-1)^{\left(\gamma, \psi_{m}(L)\right) \oplus 1} r_{K}^{L} \cdot 2^{1-m} \in \mathbb{Z}$. Без ограничения общности можно считать, что $s=m, I=\{1, \ldots, t\}$; вектор $\delta^{\prime} \in V_{m}$ представим в виде $\delta^{\prime}=\left(\delta^{\prime \prime}, \alpha\right)$, где $\delta^{\prime \prime} \in V_{m-1}, \alpha \in\{0,1\}$. Для всех $x \in H^{*}(\vec{r})$ аналогично доказательству леммы 2 легко пока- 
зать, что выполняется неравенство

$$
\begin{aligned}
& \phi_{\alpha}\left(x 2^{1-n / 2}\right)= \\
& =\sum_{\delta \in V_{m}} \frac{1}{2^{m}} \exp \left[2^{1-m} \pi i\left(\beta^{k}(\alpha), \sum_{\varnothing \neq J \subset \overline{1, m}}(-1)^{\left(\delta, \psi_{m}(J)\right) \oplus 1} r^{J}\right)\right]+O\left(2^{-5 n / 12}\right) \leq \\
& \leq \sum_{\delta \in V_{m-1}} \frac{1}{2^{m-1}}\left|\cos \left(\pi 2^{1-m}\left(\beta^{k}(\alpha), \sum_{J \subset \overline{1, m-1}}(-1)^{\left(\delta, \psi_{m-1}(J)\right)} r^{J \cup\{m\}}\right)\right)\right|+ \\
& +O\left(2^{-5 n / 12}\right) \leq 1-2^{1-m}+2^{1-m} \times \\
& \times \cos \left(\pi 2^{1-m}\left(\beta^{k}(\alpha), \sum_{J \subset \overline{1, m-1}}(-1)^{\left(\delta^{\prime \prime}, \psi_{m-1}(J)\right)} r^{J \cup\{m\}}\right)\right)+O\left(2^{-5 n / 12}\right) .
\end{aligned}
$$

Разобьем $V_{n}$ на $2^{n-t}$ групп векторов с одинаковыми последними $n-t$ координатами. Покажем, что в группе с произвольным вектором $\gamma^{\prime}$ последних $n-t$ координат найдется вектор $\alpha$ такой, что выражение $2^{1-m}\left(\beta^{k}(\alpha), \sum_{J \subset \overline{1, m-1}}(-1)^{\left(\delta^{\prime \prime}, \psi_{m-1}(J)\right)} r^{J \cup\{m\}}\right)$ не является целым.

Для этого рассмотрим величины

$$
\begin{gathered}
W_{0}^{\delta^{\prime \prime}}\left(\alpha_{1}, \ldots, \alpha_{t}\right)=2^{1-m}\left(\beta^{k}(\alpha), \sum_{J \subset \overline{1, m-1}}(-1)^{\left(\delta^{\prime \prime}, \psi_{m-1}(J)\right)} r^{J \cup\{m\}}\right)= \\
=2^{1-m}\left(\beta^{k}\left(\alpha_{1}, \ldots, \alpha_{t}, \gamma^{\prime}\right), \sum_{J \subset \overline{1, m-1}}(-1)^{\left(\delta^{\prime \prime}, \psi_{m-1}(J)\right)} r^{J \cup\{m\}}\right), \\
U_{1}\left(\alpha_{2}, \ldots, \alpha_{t}\right)=W_{0}^{\delta^{\prime \prime}}\left(1, \alpha_{2}, \ldots, \alpha_{t}\right)-W_{0}^{\delta^{\prime \prime}}\left(0, \alpha_{2}, \ldots, \alpha_{t}\right) \\
U_{2}\left(\alpha_{3}, \ldots, \alpha_{t}\right)=U_{1}\left(1, \alpha_{3}, \ldots, \alpha_{t}\right)-U_{1}\left(0, \alpha_{3}, \ldots, \alpha_{t}\right) \\
\ldots \\
U_{t}(\varnothing)=U_{t-1}(1)-U_{t-1}(0) .
\end{gathered}
$$

Предположим противное. Тогда $U_{t}(\varnothing)$ как линейная комбинация целых чисел также должна быть целой. 
С другой стороны,

$W_{0}^{\delta}\left(\alpha_{1}, \ldots, \alpha_{t}\right)=$

$=2^{1-m}\left(\sum_{J \subset \overline{1, m-1}}(-1)^{\left(\delta^{\prime \prime}, \psi_{m-1}(J)\right)} \sum_{K \subset \overline{1, n},|K| \leq k} \beta_{K}\left(\alpha_{1}, \ldots, \alpha_{t}, \gamma^{\prime}\right) \cdot r_{K}^{J \cup\{m\}}\right)$,

$U_{1}\left(\alpha_{2}, \ldots, \alpha_{t}\right)=$

$=2^{1-m}\left(\sum_{J \subset \overline{1, m-1}}(-1)^{\left(\delta^{\prime \prime}, \psi_{m-1}(J)\right)} \sum_{\substack{K \subset \overline{1, n}, K \supset\{1\},|K| \leq k}} \beta_{K}\left(\alpha_{1}, \ldots, \alpha_{t}, \gamma^{\prime}\right) \cdot r_{K}^{J \cup\{m\}}\right)$,

$U_{t}(\varnothing)=$

$$
\begin{aligned}
& =2^{1-m}\left(\sum_{J \subset \overline{1, m-1}}(-1)^{\left(\delta^{\prime \prime}, \psi_{m-1}(J)\right)} \sum_{\substack{K \subset \overline{1, n},|K| \leq k, K \supset\{1, \ldots, t\}}} \beta_{K}\left(\alpha_{1}, \ldots, \alpha_{t}, \gamma^{\prime}\right) \cdot r_{K}^{J \cup\{m\}}\right)= \\
& =\sum_{J \subset \overline{1, m-1}}(-1)^{\left(\delta^{\prime \prime}, \psi_{m-1}(J)\right)} r_{\{1, \ldots, t\}}^{J \cup\{m\}} \cdot 2^{1-m}+ \\
& +\sum_{\substack{K \subset \overline{1, n}, K \supset\{1, \ldots, t\}, t<|K| \leq k}} \sum_{J \subset \overline{1, m-1}}(-1)^{\left(\delta^{\prime \prime}, \psi_{m-1}(J)\right)} r_{K}^{J \cup\{m\}} \cdot 2^{1-m} .
\end{aligned}
$$

Так как верно равенство

$$
\sum_{J \subset \overline{1, m}, m \in J}(-1)^{\left(\delta^{\prime}, \psi_{m}(J)\right) \oplus 1} r_{I}^{J} \cdot 2^{1-m}=(-1)^{\alpha} \sum_{J \subset \overline{1, m}-1}(-1)^{\left(\delta^{\prime \prime}, \psi_{m-1}(J)\right) \oplus 1} r_{I}^{J} \cdot 2^{1-m},
$$

то из определения множества $R_{t}(m, N)$ следует, что для всех $K$ таких, что $|K|>t, \sum_{J \subset 1, m-1}(-1)^{\left(\delta, \psi_{m-1}(J)\right)} r_{K}^{J \cup\{m\}} \cdot 2^{1-m} \in \mathbb{Z}$, a сумма $\sum_{J \subset \overline{1, m-1}}(-1)^{\left(\delta^{\prime \prime}, \psi_{m-1}(J)\right)} r_{\{1, \ldots, t\}}^{J \cup\{m\}} \cdot 2^{1-m}$ не принадлежит $\mathbb{Z}$. 
Следовательно, $U_{t}(\varnothing) \notin \mathbb{Z}$, а это противоречит предположению.

Следовательно,

$$
\phi_{\alpha}\left(x 2^{1-n / 2}\right) \leq 1-2^{1-m}\left(\frac{\pi}{2^{m}}\right)^{2}+O\left(2^{-5 n / 12}\right) \leq 1-2^{-3 m} \pi^{2}
$$

Проводя далее доказательство аналогично доказательству леммы 2, легко получаем, что

$$
\left|J_{1}(\vec{r})\right| \leq V\left(H^{*}(\vec{r})\right) \exp \left(-2^{n / 7}\right)
$$

Таким образом, для всех $\vec{r} \in R_{t}(m, N)$ верно неравенство

$$
\begin{aligned}
&\left|\int_{H(\vec{r})} \phi_{\xi}\left(x 2^{1-n / 2}\right) \exp [-i(x, z)] d t\right| \\
&=\left|J_{1}(\vec{r})+J_{2}(\vec{r})\right| \leq\left(2^{n / 2} \pi\right)^{N} \exp \left(-2^{n / 7}\right) .
\end{aligned}
$$

Теперь рассмотрим векторы $\vec{r} \in \mathfrak{R}^{* *}=\mathfrak{R}^{* *}(m, N)$.

Лемма 4. Если обозначить $S^{\delta}(\vec{r}, I)=\sum_{\varnothing \neq J \subset \overline{1, m}}(-1)^{\left(\delta, \psi_{m}(J)\right) \oplus 1} r_{I}^{J} \cdot 2^{1-m}$, то вектор $\vec{r}$ принадлежст множеству $\mathfrak{R}^{* *}$ тогда и только тогда, когда для любого множества $I \subset \overline{1, n}$, для любых векторов $\delta_{1}, \delta_{2} \in V_{m}$ выполняется условие $S^{\delta_{1}}(\vec{r}, I)-S^{\delta_{2}}(\vec{r}, I) \in 2 \mathbb{Z}$.

Доказательство. Сперва докажем, что если $\vec{r} \in \mathfrak{R}^{* *}, I \subset \overline{1, n}$ и $\delta_{1}, \delta_{2} \in$ $V_{m}$, то $S^{\delta_{1}}(\vec{r}, I)-S^{\delta_{2}}(\vec{r}, I) \in 2 \mathbb{Z}$.

Разобьем все множество $V_{m}$ на множества $V^{\{k\}}=\left\{\delta \in V_{m}:\|\delta\|=k\right\}$, где $\|\delta\|$ - вес двоичного вектора $\delta, k \in\{0, \ldots, m\}$. Покажем, что если $\delta_{1} \in V^{\{k\}}, \delta_{2} \in V^{\{k-1\}}, \quad \delta_{1} \oplus \delta_{2}=e_{s}$, где $e_{s} \in V_{m}$ - двоичный вектор, $s$-й координатой которого является единица, а остальными - нули то $S^{\delta_{1}}(\vec{r}, I)-S^{\delta_{2}}(\vec{r}, I) \in 2 \mathbb{Z}$. Пусть без ограничения общности $s=m$, 
тогда

$$
\begin{aligned}
& S^{\delta_{1}}(\vec{r}, I)-S^{\delta_{2}}(\vec{r}, I)= \\
&= \sum_{\varnothing \neq J \subset \overline{1, m}, m \notin J}\left((-1)^{\left(\delta_{2}, \psi_{m}(J)\right)}-(-1)^{\left(\delta_{1}, \psi_{m}(J)\right)}\right) r_{I}^{J} \cdot 2^{1-m}+ \\
& \quad+\sum_{J \subset \overline{1, m}, m \in J}\left((-1)^{\left(\delta_{2}, \psi_{m}(J)\right)}-(-1)^{\left(\delta_{1}, \psi_{m}(J)\right)}\right) r_{I}^{J} \cdot 2^{1-m} .
\end{aligned}
$$

Если $m \notin J$, то $\left(\delta_{2}, \psi_{m}(J)\right)=\left(\delta_{1}, \psi_{m}(J)\right)$, и тогда

$$
\begin{aligned}
& S^{\delta_{1}}(\vec{r}, I)-S^{\delta_{2}}(\vec{r}, I)= \\
& =\sum_{J \subset \overline{1, m}, m \in J}(-1)^{\left(\delta_{2}, \psi_{m}(J)\right)}\left(1-(-1)^{\left(e_{m}, \psi_{m}(J)\right)}\right) r_{I}^{J} \cdot 2^{1-m}= \\
& =2 \cdot \sum_{J \subset \overline{1, m}, m \in J}(-1)^{\left(\delta_{2}, \psi_{m}(J)\right)} r_{I}^{J} \cdot 2^{1-m} .
\end{aligned}
$$

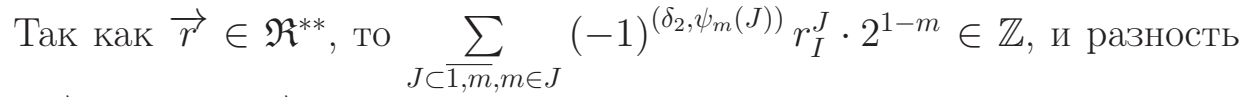
$S^{\delta_{1}}(\vec{r}, I)-S^{\delta_{2}}(\vec{r}, I) \in 2 \mathbb{Z}$.

Последовательно рассматривая $k$ от 1 до $m$, получаем что это равенство верно для любых $\delta_{1}, \delta_{2} \in V_{m}$.

Пусть теперь $S^{\delta_{1}}(\vec{r}, I)-S^{\delta_{2}}(\vec{r}, I) \in 2 \mathbb{Z}$ для любых $I \subset \overline{1, n}, \delta_{1}, \delta_{2} \in$ $V_{m}$. Покажем, что для любых $s \in \overline{1, m}, \delta^{\prime} \in V_{m}$

$$
\sum_{J \subset \overline{1, m}, s \in J}(-1)^{\left(\delta^{\prime}, \psi_{m}(J)\right) \oplus 1} r_{I}^{J} \cdot 2^{1-m} \in \mathbb{Z} .
$$

Пусть без ограничения общности $s=m, \delta^{\prime}=\left(\delta^{\prime \prime}, \alpha\right)$, где $\delta^{\prime} \in V_{m-1}$, $\alpha \in\{0,1\}$. Пусть $\delta_{1}=\left(\delta^{\prime \prime}, 0\right), \delta_{2}=\left(\delta^{\prime \prime}, 1\right)$, тогда

$$
\begin{aligned}
& S^{\delta_{1}}(\vec{r}, I)-S^{\delta_{2}}(\vec{r}, I)= \\
& =\sum_{J \subset \overline{1, m}, m \in J}(-1)^{\left(\delta_{1}, \psi_{m}(J)\right) \oplus 1}\left(1-(-1)^{\left(e_{m}, \psi_{m}(J)\right)}\right) r_{I}^{J} \cdot 2^{1-m}= \\
& =2(-1)^{\alpha} \sum_{J \subset \overline{1, m}, m \in J}(-1)^{\left(\delta^{\prime}, \psi_{m}(J)\right) \oplus 1} r_{I}^{J} \cdot 2^{1-m} \in 2 \mathbb{Z} .
\end{aligned}
$$

Следовательно, $\sum_{J \subset \overline{1, m}, s \in J}(-1)^{\left(\delta^{\prime}, \psi_{m}(J)\right) \oplus 1} r_{I}^{J} \cdot 2^{1-m} \in \mathbb{Z}$. 
Лемма 5. Для всех $\vec{r} \in \mathfrak{R}^{* *}$

$$
J_{2}(\vec{r})=J_{2}(\overrightarrow{0}) \times \exp \left[-i\left(\pi \vec{r}, z 2^{n / 2}\right)\right] .
$$

Доказательство. В обозначениях, введенных перед леммой 3, для всех $x \in H^{*}(\vec{r})$ выполняется равенство

$$
\begin{aligned}
& \phi_{\alpha}\left(x 2^{1-n / 2}\right)= \\
& =\sum_{\delta \in V_{m}} \frac{1}{2^{m}} \exp \left[2^{1-m} \pi i\left(\beta^{k}(\alpha), \sum_{\varnothing \neq J \subset \overline{1, m},|J| \leq k}(-1)^{\left(\delta, \psi_{m}(J)\right) \oplus 1} r^{J}\right)\right] \times \\
& \times \exp \left[i \sum_{\varnothing \neq J \subset \overline{1, m}}(-1)^{\left(\delta, \psi_{m}(J)\right) \oplus 1} d_{\alpha}^{J}\right]= \\
& =\sum_{\delta \in V_{m}} \frac{1}{2^{m}} \exp \left[i \sum_{\varnothing \neq J \subset \overline{1, m}}(-1)^{\left(\delta, \psi_{m}(J)\right) \oplus 1} d_{\alpha}^{J}\right] \times \\
& \times \exp \left[\pi i\left(\sum_{I \subset \overline{1, n}|I| \leq k} \sum_{\varnothing \neq J \subset \overline{1, m}}(-1)^{\left(\delta, \psi_{m}(J)\right) \oplus 1} \beta_{I}(\alpha) r_{I}^{J} \cdot 2^{1-m}\right)\right]= \\
& =\sum_{\delta \in V_{m}} \frac{1}{2^{m}} \exp \left[i \sum_{\substack{\varnothing \neq J \subset \overline{1, m} \\
|J| \leq k}}(-1)^{\left(\delta, \psi_{m}(J)\right) \oplus 1} d_{\alpha}^{J}\right] \times \\
& \times \prod_{\substack{I \subset 1, n \\
|I| \leq k}}\left(\exp \left[-\pi i \sum_{\varnothing \neq J \subset \overline{1, m}} r_{I}^{J} \cdot 2^{1-m}\right]\right)^{\beta_{I}(\alpha)} \text {. }
\end{aligned}
$$

Последнее равенство следует из леммы 4. Следовательно,

$$
\begin{gathered}
\phi_{\alpha}\left(x 2^{1-n / 2}\right)=\prod_{I \subset \overline{1, n},|I| \leq k}\left(\exp \left[-\pi i \sum_{\varnothing \neq J \subset \overline{1, m}} r_{I}^{J} \cdot 2^{1-m}\right]\right)^{\beta_{I}(\alpha)} \times \\
\times \sum_{\delta \in V_{m}} \frac{1}{2^{m}} \exp \left[i \sum_{\varnothing \neq J \subset \overline{1, m}}(-1)^{\left(\delta, \psi_{m}(J)\right) \oplus 1} d_{\alpha}^{J}\right] .
\end{gathered}
$$


Так как $r_{I}^{J} \in\left\{0,1, \ldots, 2^{m-1}-1\right\}, \quad$ то существует такое $t(I, \vec{r}) \in\left\{0,1, \ldots, 2^{m}-1\right\}, \quad$ что $\exp \left(-\pi i \sum_{\varnothing \neq J \subset \overline{1, m}} r_{I}^{J} \cdot 2^{1-m}\right)=$ $\exp \left(i \pi 2^{1-m} \cdot t(I, \vec{r})\right)$. Из определения величины $d_{\alpha}^{J}$ следует, что

$$
\sum_{\delta \in V_{m}} \frac{1}{2^{m}} \exp \left[i \sum_{\varnothing \neq J \subset \overline{1, m}}(-1)^{\left(\delta, \psi_{m}(J)\right) \oplus 1} d_{\alpha}^{J}\right]=\phi_{\alpha}\left(\left(x-\pi \cdot 2^{\frac{n}{2}} \vec{r}\right) 2^{1-\frac{n}{2}}\right) .
$$

Тогда

$$
\phi_{\alpha}\left(x 2^{1-n / 2}\right)=\phi_{\alpha}\left(\left(x-\pi \cdot 2^{\frac{n}{2}} \vec{r}\right) 2^{1-\frac{n}{2}}\right) \prod_{I \subset \overline{1, n},|I| \leq k}\left(\exp \left(i \pi 2^{1-m} \cdot t(I, \vec{r})\right)\right)^{\beta_{I}(\alpha)} .
$$

Следовательно, для всех $x \in H^{*}(\vec{r})$ верно равенство

$$
\begin{aligned}
& \phi_{\xi}\left(x 2^{1-n / 2}\right)=\prod_{\alpha \in V_{n}} \phi_{\alpha}\left(x 2^{1-n / 2}\right)= \\
= & \prod_{I \subset \frac{1, n}{1, I I}(\leq k}\left(\exp \left(i \pi 2^{1-m} \cdot t(I, \vec{r})\right)\right)^{\sum_{\alpha \in V_{n}} \beta_{I}(\alpha)} \prod_{\alpha \in V_{n}} \phi_{\alpha}\left(\left(x-\pi \cdot 2^{\frac{n}{2}} \vec{r}\right) 2^{1-\frac{n}{2}}\right)= \\
= & \phi_{\xi}\left(\left(x-\pi \cdot 2^{n / 2} \vec{r}\right) 2^{-n / 2}\right) \cdot \prod_{I \subset \overline{1, n}, I \mid \leq k}\left(\exp \left(i \pi 2^{n+1-|I|-m} \cdot t(I, \vec{r})\right)\right) .
\end{aligned}
$$

Так как $|I|=o(\sqrt{n}), m=o(n)$, то $i \pi 2^{n+1-|I|-m} \cdot t(I, \vec{r})=2 i \pi \cdot j$, где $j \in \mathbb{Z}$, и, следовательно,

$$
\phi_{\xi}\left(x 2^{1-n / 2}\right)=\phi_{\xi}\left(\left(x-\pi \cdot 2^{n / 2} \vec{r}\right) 2^{1-n / 2}\right) .
$$

Теперь рассмотрим интеграл

$$
\begin{aligned}
& J_{2}(\vec{r})=\int_{x \in H^{*}(\vec{r})} \phi_{\xi}\left(x 2^{1-n / 2}\right) \exp [-i(x, z)] d x= \\
& =\exp \left[-i\left(\pi 2^{n / 2} \vec{r}, z\right)\right] \int_{y \in H^{*}(\overrightarrow{0})} \phi_{\xi}\left(y 2^{1-n / 2}\right) \exp [-i(y, z)] d y= \\
& =J_{2}(\overrightarrow{0}) \times \exp \left[-i\left(\pi 2^{n / 2} \vec{r}, z\right)\right] .
\end{aligned}
$$


Лемма 6. Для $x \in H^{*}(\overrightarrow{0})$ все величины $d_{\alpha}^{J}=\left(\beta^{k}(\alpha), 2^{1-n / 2-m} x^{J}\right)$ по модулю не превосходят $2^{2-m-5 n / 12}$, и характеристическая функиия может быть представлена в виде

$$
\begin{array}{r}
\phi_{\xi}\left(x 2^{1-n / 2}\right)=\exp \left(-\frac{1}{2} x^{T} Q x\left(1+\tau(x)\left(2^{m}-1\right)^{4} \max _{\varnothing \neq J \in 1, m, \alpha \in V_{n}}\left|d_{\alpha}^{J}\right|^{2}\right)+\right. \\
\left.+i \theta(x) 2^{n} \max _{\varnothing \neq J \in 1, m, \alpha \in V_{n}}\left|\left(2^{m}-1\right) d_{\alpha}^{J}\right|^{3}\right)
\end{array}
$$

где Q- ковариационная матрииа случайного вектора $(\bar{w}-\mathrm{E} \bar{w}) 2^{-n / 2-m+2}, \quad|\tau(x)| \leq 12,|\theta(x)| \leq 5$ u $x^{T} Q x=$ $\sum_{\varnothing \neq J \subset \overline{1, m}} \sum_{I \subset \overline{1, n},|I| \leq k}\left(\sum_{K \subset \overline{1, n} ; K \supset I,|K| \leq k} \frac{x_{K}^{J}}{2^{|K|+m-1}}\right)^{2}$.

Доказательство. Для всех $x \in H^{*}(\overrightarrow{0})$ в обозначениях, введенных перед леммой 3 , верны равенства $x^{*}=2^{1-n / 2-m} x$, и $d_{\alpha}^{J}=$ $\left(\beta^{k}(\alpha), 2^{1-m-n / 2} x^{J}\right)$. Следовательно,

$$
\left|\left(\beta^{k}(\alpha), 2^{1-m-n / 2} x^{J}\right)\right|=\left|d_{\alpha}^{J}\right| \leq 2^{2-m-5 n / 12} .
$$

Теперь для $x \in H^{*}(\overrightarrow{0})$ рассмотрим характеристическую функцию:

$$
\begin{aligned}
\phi_{\alpha}\left(x 2^{1-\frac{n}{2}}\right)= & \sum_{\delta \in V_{m}} \frac{1}{2^{m}} \exp \left[i\left(\beta^{k}(\alpha), \sum_{\varnothing \neq J \in \overline{1, m}}(-1)^{\left(\delta, \psi_{m}(J)\right) \oplus 1} 2^{1-\frac{n}{2}-m} x^{J}\right)\right]= \\
& =\sum_{\delta \in V_{m}} \frac{1}{2^{m}} \prod_{\varnothing \neq J \subset \overline{1, m}}\left(\cos \left(d_{\alpha}^{J}\right)+i(-1)^{\left(\delta, \psi_{m}(J)\right) \oplus 1} \cdot \sin \left(d_{\alpha}^{J}\right)\right) .
\end{aligned}
$$

Упрощая данное выражение и оценивая слагаемые, в силу малости $d_{\alpha}^{J}$ легко получаем, что

$$
\begin{array}{r}
\varphi_{\alpha}\left(d_{\alpha}^{J}, \varnothing \neq J \in \overline{1, m}\right)=\left(\prod_{\varnothing \neq J \subset \overline{1, m} \cos d_{\alpha}^{J}}+\tau_{1}(x) \max _{\varnothing \neq J \in 1, m}\left|\left(2^{m}-1\right) d_{\alpha}^{J}\right|^{4}\right)+ \\
+i \theta_{1}(x) \max _{\varnothing \neq J \in \overline{1, m}}\left|\left(2^{m}-1\right) d_{\alpha}^{J}\right|^{3}
\end{array}
$$


где $\left|\tau_{1}(x)\right| \leq 2,\left|\theta_{1}(x)\right| \leq 2$.

Рассмотрим теперь $\prod_{\varnothing \neq J \in \overline{1, m}} \cos d_{\alpha}^{J}$. Так как по формуле Тейлора $\cos d_{\alpha}^{J}=1-\frac{\left(d_{\alpha}^{J}\right)^{2}}{2}+\tau(x, J)\left(d_{\alpha}^{J}\right)^{4}$, где $|\tau(x, J)| \leq 1 / 4 !=1 / 24$, то несложно показать, что

$$
\prod_{\varnothing \neq J \subset \overline{1, m}} \cos d_{\alpha}^{J}=1-\frac{1}{2} \sum_{\varnothing \neq J \subset \overline{1, m}}\left(d_{\alpha}^{J}\right)^{2}+\tau_{2}(x) \max _{\varnothing \neq J \subset \overline{1, m}}\left|\left(2^{m}-1\right) d_{\alpha}^{J}\right|^{4}
$$

где $\left|\tau_{2}(x)\right| \leq 2$.

Следовательно,

$$
\begin{aligned}
\phi_{\alpha}\left(x 2^{1-n / 2}\right)= & \left(1-\frac{1}{2} \sum_{\varnothing \neq J \subset \overline{1, m}}\left(d_{\alpha}^{J}\right)^{2}+\tau_{3}(x) \max _{\varnothing \neq J \subset \overline{1, m}}\left|\left(2^{m}-1\right) d_{\alpha}^{J}\right|^{4}\right)+ \\
& +i \theta_{1}(x) \max _{\varnothing \neq J \subset \overline{1, m}}\left|\left(2^{m}-1\right) d_{\alpha}^{J}\right|^{3}= \\
= & \left(1-\frac{1}{2} \sum_{\varnothing \neq J \subset \overline{1, m}}\left(d_{\alpha}^{J}\right)^{2}+\tau_{4}(x) \max _{\varnothing \neq J \subset \overline{1, m}}\left|\left(2^{m}-1\right) d_{\alpha}^{J}\right|^{4}\right) \times \\
& \times \exp \left(i \theta_{2}(x) \max _{\varnothing \neq J \subset \overline{1, m}}\left|\left(2^{m}-1\right) d_{\alpha}^{J}\right|^{3}\right)
\end{aligned}
$$

где $\left|\tau_{3}(x)\right| \leq 4,\left|\tau_{4}(x)\right| \leq 5,\left|\theta_{2}(x)\right| \leq 5$.

Теперь, используя разложение натурального логарифма в ряд Маклорена, получаем

$$
\begin{gathered}
\ln \left(1-\frac{1}{2} \sum_{\varnothing \neq J \subset \overline{1, m}}\left(d_{\alpha}^{J}\right)^{2}+\tau_{4}(x) \max _{\varnothing \neq J \subset \overline{1, m}}\left|\left(2^{m}-1\right) d_{\alpha}^{J}\right|^{4}\right)= \\
=-\frac{1}{2} \sum_{\varnothing \neq J \subset \overline{1, m}}\left(d_{\alpha}^{J}\right)^{2}+\tau_{5}(x) \max _{\varnothing \neq J \subset \overline{1, m}}\left|\left(2^{m}-1\right) d_{\alpha}^{J}\right|^{4},
\end{gathered}
$$

где $\left|\tau_{5}(x)\right| \leq 6$. 
Таким образом,

$$
\begin{aligned}
& \phi_{\alpha}\left(x 2^{1-n / 2}\right)=\prod_{\alpha \in V_{n}} \phi_{\xi}\left(x 2^{1-n / 2}\right)= \\
& =\prod_{\alpha \in V_{n}}\left(1-\frac{1}{2} \sum_{\varnothing \neq J \in \overline{1, m}}\left(d_{\alpha}^{J}\right)^{2}+\tau_{4}(x) \max _{\varnothing \neq J \subset \overline{1, m}}\left|\left(2^{m}-1\right) d_{\alpha}^{J}\right|^{4}\right) \times \\
& \quad \times \exp \left(i \theta_{2}(x) \max _{\varnothing \neq J \subset \overline{1, m}}\left|\left(2^{m}-1\right) d_{\alpha}^{J}\right|^{3}\right)= \\
& =\exp \left(-\frac{1}{2} \sum_{\alpha \in V_{n}} \sum_{\varnothing \neq J \subset \overline{1, m}}\left(d_{\alpha}^{J}\right)^{2}\left(1+\tau_{6}(x)\left(2^{m}-1\right)^{4} \underset{\varnothing \neq J \subset \overline{1, m}, \alpha \in V_{n}}{\max }\left|d_{\alpha}^{J}\right|^{2}\right)\right) \times \\
& \times \exp \left(i \theta_{3}(x) \cdot 2^{n} \underset{\varnothing \neq J \subset \overline{1, m}, \alpha \in V_{n}}{\max }\left|\left(2^{m}-1\right) d_{\alpha}^{J}\right|^{3}\right),
\end{aligned}
$$

где $\left|\tau_{6}(x)\right| \leq 12,\left|\theta_{3}(x)\right| \leq 5$.

Преобразуя главный член в экспоненте с использованием доказательства леммы 2 из [3], получаем, что

$$
\begin{aligned}
\sum_{\varnothing \neq J \subset \overline{1, m}} \sum_{\alpha \in V_{n}}\left(d_{\alpha}^{J}\right)^{2}= & \sum_{\varnothing \neq J \subset \overline{1, m}} \sum_{K, L \subset \overline{1, n},|K|,|L| \leq k} x_{K}^{J} x_{L}^{J} 2^{2-2 m-|K \cup L|}= \\
& =\sum_{\varnothing \neq J \subset \overline{1, m}} \sum_{I \subset \overline{1, n},|I| \leq k}\left(\sum_{K \subset \overline{1, n}, K \supset I,|K| \leq k} \frac{x_{K}^{J}}{2^{|K|+m-1}}\right)^{2} .
\end{aligned}
$$

Из попарной независимости $\left(\psi_{m}\left(J_{1}\right), f\right)(\alpha)$ и $\left(\psi_{m}\left(J_{2}\right), f\right)(\alpha)$ при любых $\alpha \in V_{n}$ и любых $J_{1} \neq J_{2}$ следует, что ковариационная матрица $Q$ распадается на $2^{m}-1$ клеток - матриц $Q_{J}$, где $\varnothing \neq J \subset \overline{1, m}$, стоящих на главной диагонали:

$$
Q=\operatorname{Diag}\left(Q_{J}, \varnothing \neq J \subset \overline{1, m}\right)
$$

Для любого допустимого $J$ и для любых $I_{1}, I_{2}$ верно

$$
\operatorname{cov}\left(\frac{w_{I_{1}}^{J}-\mathrm{E} w_{I_{1}}^{J}}{2^{n / 2+m-2}}, \frac{w_{I_{2}}^{J}-\mathrm{E} w_{I_{2}}^{J}}{2^{n / 2+m-2}}\right)=\mathrm{E} \frac{\left(w_{I_{1}}^{J}-\mathrm{E} w_{I_{1}}^{J}\right)\left(w_{I_{2}}^{J}-\mathrm{E} w_{I_{2}}^{J}\right)}{2^{n+2 m-4}}=
$$




$$
\begin{array}{r}
=2^{4-n-2 m} \mathrm{E}\left(\sum_{\alpha \in V_{n}} \beta_{I_{1}}(\alpha)\left(f^{J}(\alpha)-1 / 2\right)\right)\left(\sum_{\alpha \in V_{n}} \beta_{I_{2}}(\alpha)\left(f^{J}(\alpha)-1 / 2\right)\right)= \\
=2^{2-n-2 m} \sum_{\alpha \in V_{n}} \beta_{I_{1} \cup I_{2}}(\alpha)=2^{2-2 m-\left|I_{1} \cup I_{2}\right|} .
\end{array}
$$

Следовательно,

$$
x^{T} Q x=\sum_{\varnothing \neq J \subset \overline{1, m}} \sum_{\substack{K, L \subset \overline{1, n} \\|K|,|L| \leq k}} x_{K}^{J} x_{L}^{J} 2^{2-2 m-|K \cup L|}=\sum_{\varnothing \neq J \subset \overline{1, m}} \sum_{\alpha \in V_{n}}\left(d_{\alpha}^{J}\right)^{2} .
$$

Из леммы 6 следует, что для всех $x \in H^{*}(\overrightarrow{0})$ верно неравенство

$$
\left|\phi_{\xi}\left(x 2^{1-n / 2}\right)\right| \leq \exp \left(-\frac{1-2^{8+2 m-5 n / 6}}{2} x^{T} Q x\right) .
$$

Лемма 7. Во введенных ранее обозначениях справедливы равенства

$$
\begin{gathered}
\operatorname{det} Q=\exp _{2}\left(\left(2^{m}-1\right)(n-k)\left(\begin{array}{c}
n \\
k
\end{array}\right)-(n+2 m-2) N(n, m, k)\right), \\
z^{T} Q^{-1} z=2^{2 m-2} \sum_{\varnothing \neq J \subset 1, m} \sum_{\substack{I \subset 1, n \\
|I| \leq k}}\left(\sum_{K \subset I}(-1)^{|K|} 2^{|K|} z_{K}^{J}\right)^{2} .
\end{gathered}
$$

Доказательство. Пусть $y_{I}^{J}=\sum_{K \supset I} \frac{x_{K}^{J}}{2^{|K|+m-1}}$. Согласно лемме 6 для матрицы $A$ перехода от $x$ к $y(y=A x)$ справедливо равенство $x^{T} Q x=$ $y^{T}\left(A^{-1}\right)^{T} Q A^{-1} y=y^{T} y$. Следовательно, $Q=A^{T} A, Q^{-1}=A^{-1}\left(A^{-1}\right)^{T}$.

При этом матрица $A$ распадается на $2^{m}-1$ клеток - матриц $A^{J}, \varnothing \neq J \subset \overline{1, m}$, стоящих на главной диагонали, т.е. $A=$ $\operatorname{Diag}\left(A^{J}, \varnothing \neq J \subset \overline{1, m}\right)$.

Рассмотрим $J$-ю клетку размера $L(n, k) \times L(n, k)$. Элемент $A_{I K}^{J}$ равен $2^{1-|K|-m}$, если $I \subset K$, и нулю в противоположном случае, поэтому $\mathrm{A}-$ 
верхнетреугольная матрица и

$$
\begin{aligned}
\operatorname{det} Q=(\operatorname{det} A)^{2}= & \prod_{\varnothing \neq J \subset \overline{1, m}} \prod_{\substack{I \subset \overline{1, n} \\
|I| \leq k}} 2^{2-2|I|-2 m}= \\
& =\exp _{2}\left(-2 \sum_{s=1}^{m}\left(\begin{array}{c}
m \\
s
\end{array}\right) \sum_{i=0}^{k}(i+m-1)\left(\begin{array}{c}
n \\
i
\end{array}\right)\right) .
\end{aligned}
$$

Используя доказательство леммы 3 из [3], из данного выражения для $\operatorname{det} Q$ легко получить первое равенство леммы.

Рассмотрим матрицу $B=\operatorname{Diag}\left(B^{J}, \varnothing \neq J \subset \overline{1, m}\right)$. Пусть для клетки $B^{S}$ размера $L(n, k) \times L(n, k)$ элемент $b_{I K}^{S}$ равен $(-1)^{|K|-|I|} 2^{|I|+m-1}$, если $I \subset K$, и 0 в противном случае. Несложно показать, что $B=A^{-1}$. Очевидно, что

$$
\begin{gathered}
z^{T} Q^{-1} z=z^{T} A^{-1}\left(A^{-1}\right)^{T} z=z^{T} B B^{T} z= \\
=\sum_{\varnothing \neq J \subset \overline{1, m}} \sum_{\substack{I \subset \overline{1, n} \\
|I| \leq k}}\left(\sum_{K \subset I}(-1)^{|I|-|K|} 2^{|K|+m-1} z_{K}^{J}\right)^{2}= \\
=2^{2 m-2} \sum_{\varnothing \neq J \subset \overline{1, m}} \sum_{\substack{I \subset \overline{1, n} \\
|I| \leq k}}\left(\sum_{K \subset I}(-1)^{|K|} 2^{|K|} z_{K}^{J}\right)^{2} .
\end{gathered}
$$

Введем область

$$
\begin{aligned}
& H^{* *}= \\
= & \left\{x \in 2^{n / 2-1} G:\left|x_{I}^{J}\right| \leq 2^{|I|+m-1} \sqrt{n^{k+1} \ln 4}, \varnothing \neq J \subset \overline{1, m}, I \subset \overline{1, n},|I| \leq k\right\},
\end{aligned}
$$

Легко показать, что $H^{* *}$ содержится в $H^{*}(\overrightarrow{0})$. Представим интеграл $J_{2}(\overrightarrow{0})$ в виде суммы интегралов по областям $H^{*}(\overrightarrow{0}) \backslash H^{* *}$ и $H^{* *}$. Обозначим эти интегралы соответственно $J_{3}$ и $J_{4}$.

Лемма 8. Во введенных обозначениях верно следующее равенство:

$$
J_{3}=\sqrt{\frac{(2 \pi)^{N}}{\operatorname{det} Q}} O\left(n^{2 k} 2^{-\frac{2 n}{3}+k+2 m}\right) .
$$


Доказательство.

$$
\begin{aligned}
\left|J_{3}\right| \leq \int_{H^{*} \backslash H^{* *}} \exp \left(-\frac{1-2^{8+2 m-5 n / 6}}{2} x^{T} Q x\right) d x \leq \\
\quad \leq \int_{\mathbb{R}^{N \backslash H^{* *}}} \exp \left(-\frac{1-2^{8+2 m-5 n / 6}}{2} x^{T} Q x\right) d x .
\end{aligned}
$$

Если обозначить для упрощения дальнейшей записи $\mu=1-2^{8+2 m-5 n / 6}$, то очевидно, что $\left|J_{3}\right| \leq \sqrt{\frac{(2 \pi)^{N}}{\operatorname{det}(\mu Q)}} \mathrm{P}\left\{\eta \in \mathbb{R}^{N} \backslash H^{* *}\right\}$, где $\eta-$ нормально распределенный вектор с параметрами $(\overline{\mathrm{o}}, \mu Q)$.

Очевидно, что

$$
\begin{gathered}
\mathrm{P}\left\{\eta \in \mathbb{R}^{N} \backslash H^{* *}\right\} \leq \sum_{\substack{\phi \neq J \subset \overline{1, m} \\
I \subset \overline{1, n}, 0 \leq|I| \leq k}} \mathrm{P}\left\{\left|\eta_{I}^{J}\right|>2^{|I|+m-1} \sqrt{n^{k+1} \ln 4}\right\}, \\
\mathrm{P}\left\{\left|\eta_{I}^{J}\right|>2^{|I|+m} \sqrt{n^{k} \ln 4}\right\} \leq \frac{2}{\sqrt{2 \pi}} \int_{2^{|I|+m-1} \sqrt{n^{k} \ln 4}}^{+\infty} \exp \left(-\frac{s^{2}}{2 \mathrm{D} \eta_{I}^{J}}\right) d s .
\end{gathered}
$$

Используя обозначения из леммы 7 , получаем что

$$
\begin{aligned}
& \mathrm{D} \eta_{I}^{J}=\mu\left(Q^{-1}\right)_{I I}^{J}=\mu\left(A^{-1}\left(A^{-1}\right)^{T}\right)_{I I}^{J}=\mu \sum_{S \subset \overline{1, n},|S| \leq k, S \supset I}\left(b_{I S}^{J}\right)^{2}= \\
&=\mu \cdot 2^{2|I|+2 m-2} \sum_{S \subset \overline{1, n},|S|+|J| \leq k, S \supset I} 1=\mu \cdot 2^{2|I|+2 m-2} L(n-|I|, k-|I|) .
\end{aligned}
$$

В [3] показано, что $L(n, k)=O\left(n^{k}\right)$, следовательно, если обозначить $L(n-|I|, k-|I|)=L^{\prime}$, то при всех достаточно больших $n$

$$
\begin{gathered}
\int_{2^{|I|+m-1} \sqrt{n^{k} \ln 4}}^{+\infty} \exp \left(-\frac{s^{2}}{2 \mathrm{D} \eta_{I}^{J}}\right) d s=2^{|I|+m-1} \sqrt{\mu \cdot L^{\prime}} \int_{\sqrt{\frac{n^{k+1} \ln 4}{\mu L^{\prime}}}}^{+\infty} \exp \left(-\frac{t^{2}}{2}\right) d t \leq \\
\leq 2^{|I|+m-1} \sqrt{\mu \cdot L^{\prime}} \int_{\sqrt{\frac{n^{k+1} \ln 4}{\mu L^{\prime}}}}^{+\infty} t \exp \left(-\frac{t^{2}}{2}\right) d t=O\left(n^{k / 2} 2^{-\frac{2 n}{3}+m+k}\right) .
\end{gathered}
$$




$$
\left|J_{3}\right| \leq \sqrt{\frac{(2 \pi)^{N}}{\mu^{N} \operatorname{det} Q}}\left(2^{m}-1\right) L(n, k) O\left(n^{\frac{k-1}{2}} 2^{-\frac{2 n}{3}+m+k}\right)
$$

где $\mu^{N}=\left(1-2^{8+2 m-5 n / 6}\right)^{N}=1+o(1)$.

В обозначениях леммы 6 получается, что для всех $x \in H^{* *}$

$$
\left|d_{\alpha}^{J}\right| \leq \sum_{i=0}^{k}\left(\begin{array}{c}
n \\
i
\end{array}\right) 2^{1-n / 2-m} 2^{i+m-1} \sqrt{n^{k+1} \ln 4} \leq 2^{-n / 2} L(n, k) 2^{k} O\left(n^{(k+1) / 2}\right) .
$$

В [3] доказано, что $2^{k} L(n, k)=2^{k} M(n, k)=O\left(n^{k}\right)$, следовательно,

$$
\begin{gathered}
\left|d_{\alpha}^{J}\right|=O\left(2^{-n / 2} n^{(3 k+1) / 2}\right), \\
\left.x^{T} Q x=\sum_{\varnothing \neq J \subset \overline{1, m}} \sum_{\substack{|\subset \overline{1, n}\\
| I \mid \leq k}} \sum_{\substack{K \subset \overline{1, n}, K \supset I \\
|K| \leq k}} \frac{x_{K}^{J}}{2^{|K|+m-1}}\right)^{2} \leq \\
\leq 2^{m} \sum_{i=0}^{k}\left(\begin{array}{c}
n \\
i
\end{array}\right)\left(\sum_{t=0}^{k}\left(\begin{array}{l}
n \\
t
\end{array}\right) \sqrt{n^{k+1} \ln 4}\right)^{2} \leq \\
\leq 2^{m+1} n^{k+1}\left((k+1)\left(\begin{array}{l}
n \\
k
\end{array}\right)\right)^{3}=O\left(2^{m} n^{4 k+4}\right) .
\end{gathered}
$$

Значит, равномерно по $x \in H^{* *}$

$$
\begin{aligned}
& \phi_{\xi}\left(2^{1-n / 2} x\right)=\exp \left(-\frac{1}{2} x^{T} Q x\right) \exp \left(O\left(n^{5 k+4} 2^{-n+5 m}\right)\right) \times \\
& \times \exp \left(O\left(n^{3 k+3} 2^{-n / 2+3 m}\right)\right)=\exp \left(-\frac{1}{2} x^{T} Q x\right)\left(1+O\left(n^{5 k+4} 2^{-n+5 m}\right)\right) \times \\
& \times\left(1+O\left(n^{3 k+3} 2^{-n / 2+3 m}\right)\right)=\exp \left(-\frac{1}{2} x^{T} Q x\right)\left(1+O\left(n^{3 k+3} 2^{-n / 2+3 m}\right)\right) .
\end{aligned}
$$

Интеграл $J_{4}$, введенный перед леммой 8, мы можем представить в 
виде

$$
\begin{aligned}
J_{4}=\int_{\mathbb{R}^{N}} e^{-\frac{1}{2} x^{T} Q x} e^{-i(x, z)} d x & -\int_{\mathbb{R}^{N} \backslash H^{* *}} e^{-\frac{1}{2} x^{T} Q x} e^{-i(x, z)} d x+ \\
& +\int_{H^{* *}} e^{-\frac{1}{2} x^{T} Q x} O\left(n^{3 k+3} 2^{3 m+n / 2}\right) e^{-i(x, z)} d x ;
\end{aligned}
$$

обозначим интегралы в правой части через $J_{5}, J_{6}, J_{7}: J_{4}=J_{5}-J_{6}+J_{7}$.

В обозначениях, использованных при доказательстве леммы 6 , имеем:

$$
\begin{aligned}
J_{5}=\int_{\mathbb{R}^{N}} \exp \left(-\frac{1}{2} x^{T} Q x\right) \exp (-i(x, z)) d x= \\
=\frac{1}{\operatorname{det} A} \int_{\mathbb{R}^{N}} \exp \left(-i\left(y,\left(A^{-1}\right)^{T} z\right)\right) \exp \left(-\frac{1}{2} y^{t} y\right) d y= \\
=\sqrt{\frac{(2 \pi)^{N}}{\operatorname{det} Q}} \int_{\mathbb{R}^{N}} \exp \left(-i\left(y,\left(A^{-1}\right)^{T} z\right)\right) p_{\eta^{\prime}}(y) d y
\end{aligned}
$$

где $p_{\eta^{\prime}}(y)$ - плотность нормально распределенного вектора с параметрами $(\overline{\mathrm{o}}, E), \mathrm{E}-$ единичная матрица размера $N \times N$. Мы получили формулу обращения для характеристической функции многомерной нормальной величины [1, с. 168], согласно которой:

$$
J_{5}=\sqrt{\frac{(2 \pi)^{N}}{\operatorname{det} Q}} \exp \left(-\frac{1}{2} z^{T} A^{-1}\left(A^{-1}\right)^{T} z\right)=\sqrt{\frac{(2 \pi)^{N}}{\operatorname{det} Q}} \exp \left(-\frac{1}{2} z^{T} Q^{-1} z\right) .
$$

Аналогично оценке интеграла $J_{3}(\overrightarrow{0})$ в лемме 8 легко показать, что

$$
J_{6}=\int_{\mathbb{R}^{N} \backslash H^{* *}} \exp \left(-\frac{1}{2} x^{T} Q x\right) \exp (-i(x, z)) d x=\sqrt{\frac{(2 \pi)^{N}}{\operatorname{det} Q}} O\left(n^{\frac{3 k-1}{2}} 2^{-\frac{2 n}{3}+k+2 m}\right) .
$$

Оценим теперь $J_{7}$ :

$$
\begin{array}{r}
\left|J_{7}\right| \leq \int_{H^{* *}}\left|\exp \left(-\frac{1}{2} x^{T} Q x\right) O\left(n^{3 k+3} 2^{3 m+n / 2}\right) \exp (-i(x, z))\right| d x= \\
=\sqrt{\frac{(2 \pi)^{M}}{\operatorname{det} Q}} O\left(n^{3 k-3} 2^{3 m-n / 2}\right) .
\end{array}
$$


Теперь, собрав все оценки воедино, мы получаем, что

$$
\begin{gathered}
J_{2}=\int_{H^{*}(\overrightarrow{0})} \phi_{\xi}\left(x 2^{1-n / 2}\right) \exp [-i(x, z)] d x=\sqrt{\frac{(2 \pi)^{N}}{\operatorname{det} Q} \times} \\
\times\left(\exp \left(-\frac{1}{2} z^{T} Q^{-1} z\right)+O\left(n^{-1+3 k / 2} 2^{-2 n / 3+k+2 m}\right)+O\left(n^{3 k-3} 2^{-n / 2+3 m}\right)\right)= \\
=\frac{\exp \left(-\frac{1}{2} z^{T} Q^{-1} z\right)+O\left(n^{3 k-3} 2^{-n / 2+3 m}\right)}{\exp _{2}\left(\frac{(n-k)}{2}\left(\begin{array}{l}
n \\
k
\end{array}\right)\left(2^{m}-1\right)-\frac{1}{2}\left(n+2 m+\log _{2} \frac{\pi}{2}\right) N\right)} .
\end{gathered}
$$

Из этого равенства и лемм 2, 3 и 5 следует, что

$$
\begin{aligned}
& \left(2^{m-1+n / 2} \pi\right)^{N} \mathrm{P}\left(\xi=z 2^{n / 2-1}\right)= \\
& =\sum_{\vec{r} \in R(m, N) \backslash \Re^{* *}}\left(2^{n / 2} \pi\right)^{N} O\left(\exp \left(-2^{n / 7}\right)\right)+ \\
& +\sum_{\vec{r} \in \mathfrak{R}^{* *}} \int_{H(\vec{r})} \phi_{\xi}\left(x 2^{1-n / 2}\right) \exp [-i(x, z)] d t= \\
& =\int_{H^{*}(\overrightarrow{0})} \phi_{\xi}\left(x 2^{1-n / 2}\right) \exp [-i(x, z)] d t \times \sum_{\vec{r} \in \Re^{* *}} \exp \left[-i\left(\pi 2^{n / 2} \vec{r}, z\right)\right]+ \\
& +\left(2^{n / 2+m-1} \pi\right)^{N} O\left(\exp \left(-2^{n / 7}\right)\right)= \\
& =\frac{\exp \left(-\frac{1}{2} z^{T} Q^{-1} z\right)+O\left(n^{3 k-3} 2^{-n / 2+3 m}\right)}{\exp _{2}\left(\frac{(n-k)}{2}\left(\begin{array}{l}
n \\
k
\end{array}\right)\left(2^{m}-1\right)-\frac{1}{2}\left(n+2 m+\log _{2} \frac{\pi}{2}\right) N\right)} \times \\
& \times\left(\sum_{\vec{r} \in \mathfrak{R}^{* *}} \exp \left[-i\left(\pi 2^{n / 2} \vec{r}, z\right)\right]\right) .
\end{aligned}
$$

Из данного равенства и следует утверждение теоремы.

Данная теорема фактически, является предельной теоремой для сумм случайных векторов, размерность которых стремится к бесконечности вместе с числом слагаемых. Изучению таких сумм посвящена, в частности, работа [6]. Множитель, зависящий от множества $\mathfrak{R}^{* *}(m, N)$, является согласно доказательству теоремы действительнозначным. 
Согласно доказательству леммы 6 , в представлении $\phi_{\alpha}\left(x 2^{1-n / 2}\right)$ через тригонометрические функции от аргумента $d_{\alpha}^{J}$ присутствует мнимая часть только при $m \geq 2$. Если $m=1$, то равномерно по $x \in H^{* *}$ выполняется $\phi_{\xi}\left(2^{1-n / 2} x\right)=\exp \left(-\frac{1}{2} x^{T} Q x\right)\left(1+O\left(n^{5 k+4} 2^{-n}\right)\right)$ и, следовательно, если провести доказательство теоремы с учетом этого факта, и принимать во внимание, что $2^{n / 2-1} G=H(\overrightarrow{0})$, мы получим следующий результат.

Теорема 9. Пусть $n \rightarrow \infty, k(n)=o(\sqrt{n}), m=1, Q=Q(n, 1, k+1)$ - ковариачионная матрица случайного вектора ( $\bar{w}-\mathrm{E} \bar{w}) 2^{-n / 2+1}$, $\bar{z}(n) 2^{-1+n / 2}$ - последовательность иелочисленных векторов размерности $M(n, 1, k+1)$. Тогда равномерно относительно $\bar{z}(n)$ верно:

$$
\begin{gathered}
\mathrm{P}\left(\bar{w}=\mathrm{E} \bar{w}+\bar{z} 2^{n / 2-1}\right)=\frac{\exp \left(-\frac{1}{2} z^{T} Q^{-1} z\right)+O\left(n^{5 k+4} 2^{-n}\right)}{\left(2^{\frac{n-1}{2}}\right)^{M(n, 1, k+1)} \sqrt{\pi^{M(n, 1, k+1)} \operatorname{det} Q}}= \\
\frac{\exp \left(-\frac{1}{2} \sum_{I \subset 1, n,|I| \leq k}\left(\sum_{K \subset \overline{1, n}, K \subset I,|I| \leq k}(-1)^{|I|-|K|} 2^{|K|} z_{K}^{I}\right)^{2}\right)+O\left(n^{5 k+4} 2^{-n}\right)}{\exp _{2}\left(\frac{(n-k)}{2}\left(\begin{array}{l}
n \\
k
\end{array}\right)+M(n, 1, k+1) \log _{2} \sqrt{\pi / 2}\right)} .
\end{gathered}
$$

Утверждение данной теоремы, с точностью до значения суммы под знаком квадрата в экспоненте в числителе, совпадает с утверждением, доказанным в [3].

Как отмечено в [8], $(n, m, k)$-устойчивые двоичные отображения являются хорошо известным объектом в математике и ее приложениях.

Согласно критерию, доказанному в [2], ненулевая комбинация $f^{J}$ координатных функций функции $f$ будет $(n, 1, k)$-устойчивой, если для любого такого $I$, что $|I| \leq k$, верно равенство

$$
w_{I}^{J}=2^{-|I|} w_{\varnothing}^{J}=2^{-|I|}\|f\| .
$$

Множество отображений, являющихся $(n, m, k)$-устойчивыми, обозначим через $R[n, m, k]$. Любая ненулевая комбинация координатных функций этих отображений является $(n, 1, k)$-устойчивой функцией.

Докажем асимптотическую формулу, являющуюся обобщением результата из [3]. 
Утверждение 10. Пусть $n \rightarrow \infty, k(n)=o(\sqrt{n}), m(n)=o(n)$. Тогда

$$
\begin{aligned}
& |R[n, m, k]| \sim\left|\mathfrak{R}^{* *}(m, N)\right| \times \\
& \quad \times \exp _{2}\left(m 2^{n}-\frac{(n-k)}{2}\left(\begin{array}{l}
n \\
k
\end{array}\right)\left(2^{m}-1\right)-N(n, m, k) \log _{2} \sqrt{\pi / 2}\right),
\end{aligned}
$$

где множество $\mathfrak{R}^{* *}(m, N)$ определено в формулировке теоремы 1

Доказательство. Используя критерий из [2], легко видеть, что верна следующая цепочка равенств:

$$
\begin{aligned}
\frac{|R[n, m, k]|}{\left|B_{n}^{m}\right|}= & \mathrm{P}\{f \in R[n, m, k]\}= \\
& =\mathrm{P}\left\{\forall J, I: 0 \leq|I| \leq k, w_{I}^{J}=2^{n-|I|-1}\right\}=\mathrm{P}\{\bar{w}=\mathrm{E} \bar{w}\} .
\end{aligned}
$$

Мы попадаем в условия теоремы 1. Из нее следует, что

$$
\begin{array}{r}
\mathrm{P}(\bar{w}=\mathrm{E} \bar{w})=\exp _{2}\left(-\frac{(n-k)}{2}\left(\begin{array}{l}
n \\
k
\end{array}\right)\left(2^{m}-1\right)-N(n, m, k) \log _{2} \sqrt{\pi / 2}\right) \times \\
\times\left(1+O\left(n^{3 k+3} 2^{-n / 2+3 m}\right)\right)\left|\mathfrak{R}^{* *}(m, N)\right| .
\end{array}
$$

Осталось заметить, что $O\left(n^{3 k+3} 2^{-n / 2+3 m}\right)=O(1)$ при $n \rightarrow \infty$.

Так как $\left|\Re^{* *}(1, N)\right|=1$, то при $m=1$ из утверждения 10 следует асимптотическая оценка для числа $(n, 1, k)$-устойчивых булевых функций, совпадающая с утверждением, доказанным в [3].

Справедливо следующее утверждение.

Следствие 11. Пусть $n \rightarrow \infty, k(n)=o(\sqrt{n}), m \in\{2,3,4\}$. Тогда

$$
\begin{aligned}
|R[n, m, k]| \sim \exp _{2}\left(2^{n}-\frac{n-k}{2}\left(\begin{array}{l}
n \\
k
\end{array}\right)\left(2^{m}-1\right)-N(n, m, k) \log _{2} \sqrt{\pi / 2}\right) \\
\times \exp _{2}\left(N(n, 1, k)\left(4 m^{2}-16 m+17\right)\right) .
\end{aligned}
$$

Вопрос о том, чему равен множитель, зависящий от множества $\mathfrak{R}^{* *}(m, N)$ в формулировке теоремы 1 , или чему равна мощность этого множества, в общем случае остается открытым. 


\section{Список литературы}

[1] Боровков A. А. Теория вероятностей. М.: УРСС, 2009, 656 с.

[2] Денисов О. В. Асимптотическая формула для числа корреляционноиммунных порядка $k$ булевых функций // Дискретная математика. - 1991. - Т. 3. Вып. 2. - С. 25-46.

[3] Денисов О. В. Локальная предельная теорема для распределения части спектра случайной двоичной функции // Дискретная математика. - 2000. - Т.12. Вып.1. - С. 82-95.

[4] Логачев О.А., Сальников А.А., Смышляев С.В., Ященко В.В. Булевы функции в теории кодирования и криптологии. - М.: МЦНМО, 2012. - 584 c.

[5] Панков К.Н. Оценки скорости сходимости в предельных теоремах для совместных распределений части характеристик случайных двоичных отображений // Прикладная дискретная математика. - 2012. - Т. 5. Вып. 4. - С. 14-30.

[6] Прохоров Ю.В. Предельные теоремы для сумм случайных векторов, размерность которых стремится к бесконечности // Теория вероятн. и ее примен. - 1990. - Т. 35. Вып. 4. - С. 751-753.

[7] Севастьянов Б.А. Курс теории вероятностей и математической статистики. - М. : Наука, 1982. - 256 с.

[8] Таранников Ю.В. О корреляционно-иммунных и устойчивых булевых функциях // Матем. вопросы кибернетики. - 2002. Вып. 11. C. $91-148$.

[9] Ященко В.В. Свойства булевых отображений, сводимые к свойствам их координатных функций // Вестник МГУ, сер. Математика. 1997. - Т. 33. №1. - C. 11-13.

[10] Carlet C. Vectorial Boolean functions for cryptography // Boolean Models and Methods in Mathematics, Computer Science, and Engineering. Encyclopedia of Mathematics and its Applications. Vol. 134. - New York: Cambridge University Press, 2010. - 780 p.

[11] Canfield E. R., Gao Z., Greenhill C., McKay B. D., Robinson R. W. Asymptotic enumeration of correlation-immune Boolean functions Cryptography and Communications. - 2010. - Vol. 2. №1. - P. 111-126. 